\title{
Structural characterization of the phospholipid stabilizer layer at the solid-liquid interface of dispersed triglyceride nanocrystals with small-angle $x$-ray and neutron scattering
}

\author{
Martin Schmiele, ${ }^{1}$ Torben Schindler, ${ }^{1}$ Tobias Unruh,,${ }^{1, *}$ Sebastian Busch, ${ }^{2}$ Humphrey Morhenn, ${ }^{3}$ Martin Westermann, ${ }^{4}$ \\ Frank Steiniger, ${ }^{4}$ Aurel Radulescu, ${ }^{5}$ Peter Lindner, ${ }^{6}$ Ralf Schweins, ${ }^{6}$ and Peter Boesecke ${ }^{7}$ \\ ${ }^{1}$ Professur für Nanomaterialcharakterisierung (Streumethoden), Friedrich-Alexander-Universität Erlangen-Nürnberg, \\ Staudtstrasse 3, 91058 Erlangen, Germany \\ ${ }^{2}$ Department of Biochemistry, University of Oxford, South Parks Road, Oxford OX1 3QU, United Kingdom \\ ${ }^{3}$ Forschungs-Neutronenquelle Heinz, Maier-Leibnitz, (FRM II), Lichtenbergstrasse 1, 85747 Garching, Germany \\ ${ }^{4}$ Center for Electron Microscopy of the Jena University Hospital, Ziegelmühlenweg 1, 07743 Jena, Germany \\ ${ }^{5}$ Jülich Centre for Neutron Science (JCNS), Lichtenbergstrasse 1, 85747 Garching, Germany \\ ${ }^{6}$ DS/LSS, Institut Laue-Langevin (ILL), 6 rue Jules Horowitz, 38042 Grenoble CEDEX 9, France \\ ${ }^{7}$ European Synchrotron Radiation Facility (ESRF), 6 rue Jules Horowitz, 38042 Grenoble CEDEX 9, France
}

(Received 31 January 2013; published 20 June 2013)

\begin{abstract}
Dispersions of crystalline nanoparticles with at least one sufficiently large unit cell dimension can give rise to Bragg reflections in the small-angle scattering range. If the nanocrystals possess only a small number of unit cells along these particular crystallographic directions, the corresponding Bragg reflections will be broadened. In a previous study of phospholipid stabilized dispersions of $\beta$-tripalmitin platelets [Unruh, J. Appl. Crystallogr. 40, 1008 (2007)], the x-ray powder pattern simulation analysis (XPPSA) was developed. The XPPSA method facilitates the interpretation of the rather complicated small-angle $\mathrm{X}$-ray scattering (SAXS) curves of such dispersions of nanocrystals. The XPPSA method yields the distribution function of the platelet thicknesses and facilitates a structural characterization of the phospholipid stabilizer layer at the solid-liquid interface between the nanocrystals and the dispersion medium from the shape of the broadened 001 Bragg reflection. In this contribution an improved and extended version of the XPPSA method is presented. The SAXS and small-angle neutron scattering patterns of dilute phospholipid stabilized tripalmitin dispersions can be reproduced on the basis of a consistent simulation model for the particles and their phospholipid stabilizer layer on an absolute scale. The results indicate a surprisingly flat arrangement of the phospholipid molecules in the stabilizer layer with a total thickness of only $12 \AA$. The stabilizer layer can be modeled by an inner shell for the fatty acid chains and an outer shell including the head groups and additional water. The experiments support a dense packing of the phospholipid molecules on the nanocrystal surfaces rather than isolated phospholipid domains.
\end{abstract}

DOI: 10.1103/PhysRevE.87.062316

PACS number(s): 82.70.Kj, 61.05.cf, 61.05.fg, 68.08.De

\section{INTRODUCTION}

For 20 years phospholipid stabilized triglyceride and, in particular, tripalmitin suspensions have been studied as potential drug delivery systems in pharmaceutical research [1-3].

After their production in the molten state, the liquid triglyceride nanodroplets crystallize upon cooling. Subsequently, they transform from rather spherical triglyceride nanoparticles in their $\alpha$ phase into thin crystalline platelets in the thermodynamically stable $\beta$ modification (cf. Fig. 1). This is accompanied by a strong increase in the particle surface, promoting gelation of the dispersion [4] unless further costabilizers like sodium glycocholate $(\mathrm{NaGC})$ are added.

Several studies have been conducted to elucidate the mechanism of phospholipid stabilized solid triglyceride suspension gelation. It is assumed that the excess phospholipids that do not adhere to the emulsion droplets aggregate in vesicles which cannot cover the freshly created particle during crystallization because of their slow diffusion [3-5]. The costabilizer NaGC is assumed to increase the speed of the surface covering, either directly due to its larger diffusion coefficient or by disrupting the vesicular structure so that the phospholipids can diffuse faster [3-5].

*Corresponding author: Tobias.Unruh@fau.de
Small-angle $\mathrm{x}$-ray scattering patterns of suspensions of $\beta$ triglyceride nanoparticles exhibit a strongly broadened 001 Bragg reflection due to the small thickness of the plateletlike crystals. For concentrations above $4 \mathrm{wt} \%$ it was found that the platelets start to self-assemble into stacks [6,7]. This can be observed for example from the interference maxima in the low angle range of the small-angle $\mathrm{x}$-ray and neutron scattering (SAXS, SANS) patterns in Fig. 2 of concentrated (10wt $\%$ ) tripalmitin suspensions stabilized with the phospholipid blend Lipoid S100 or DLPC. Especially in the case of the DLPC stabilized suspension, these interparticle interferences lead to a significant deformation of tripalmitin's 001 Bragg reflection.

The scattering patterns of isotropically scattering nanoparticle-containing dispersions that are homogeneous on large length scales, i.e., in the limit of small $Q$, are typically calculated as the product of a particle-specific form factor $P(Q)$ and a structure factor $S(Q)$ that describes interparticle interferences. Expressions of $P(Q)$ for numerous (coreshell-)particle geometries and for different kinds of interactions for $S(Q)$ can be found elsewhere [8] and are implemented in standard small-angle scattering data analysis packages like IRENA [9] or SASFIT [10]. For crystalline nanoparticles this approach is possible only within certain limits. Only if the Bragg reflections are sharp enough (i.e., the corresponding crystallographic length long enough) can analytical peak shape 


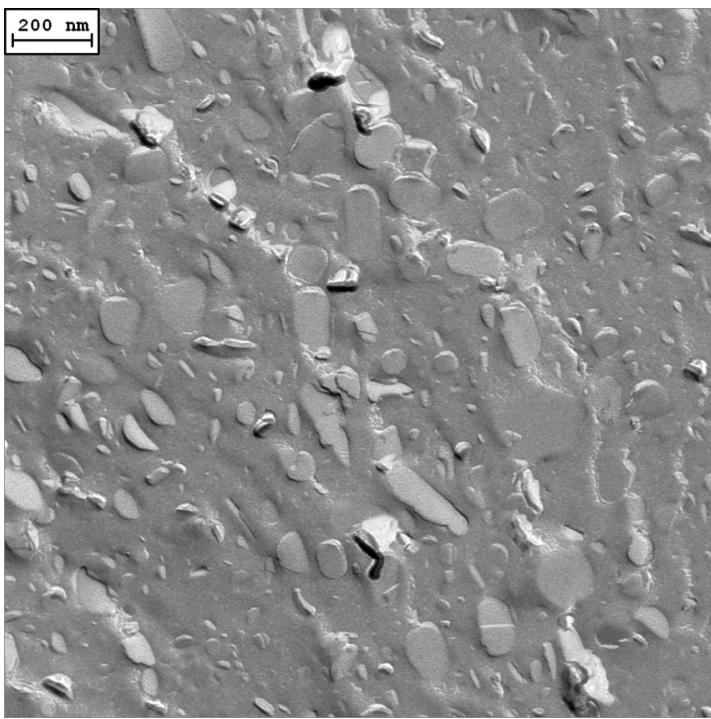

FIG. 1. Freeze fracture electron micrograph taken of a tripalmitin suspension with S100 (10\% tripalmitin, $2.4 \%$ S100, 0.6\% NaGC, in $\mathrm{H}_{2} \mathrm{O}$ ) showing the characteristic thin platelets of the $\beta$-triglyceride nanoparticles.

functions be included in $S(Q)$ to describe the scattering patterns. Employing Scherrer's equation [11] on the peak widths, it is then possible to estimate the average thickness of the platelets, but not the corresponding distribution function.

This approach will fail for crystalline nanoparticles with broad Bragg reflections because of complicated interference effects between the scattering due to the internal crystalline particle structure, the phospholipid stabilizer layer, the particle arrangement, and the overall particle shape [12]. Therefore, the $\mathrm{x}$-ray powder pattern simulation analysis (XPPSA) has been

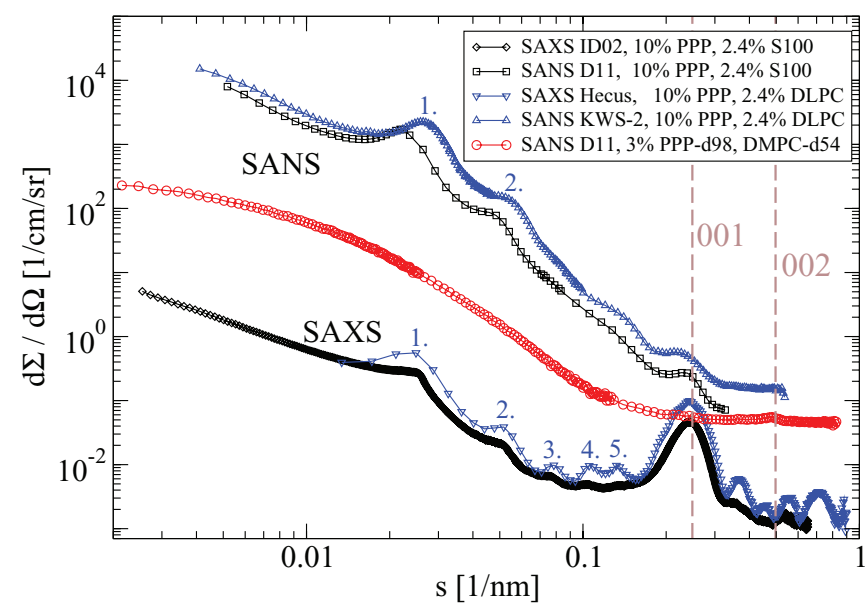

FIG. 2. (Color online) SAXS and SANS patterns for concentrated $(10 \mathrm{wt} \%)$ tripalmitin suspensions stabilized by $\mathrm{S} 100$ (black $\diamond$ and $\square$ ) or DLPC (blue $\nabla$ and $\triangle$ ). Dashed lines mark the positions of the 001 and 002 Bragg reflections for bulk $\beta$-tripalmitin. For the suspension with DLPC, the first five (two) orders of the inter-particle interference maxima in the SAXS (SANS) data are labeled by numbers. The red (०) SANS curve stems from a dilute suspension with deuterated tripalmitin-d98 stabilized with chain-deuterated DMPC-d54. For better clarity, the black ( $\square$ and $\diamond$ ) and blue $(\nabla)$ curves were divided by $1.5,10$ and 5 , respectively. developed [12] to reproduce the complicated SAXS patterns of these suspensions on the basis of a distinct structural model. It was found that the broadened 001 Bragg reflection is very sensitive to the thicknesses and scattering contrasts in the phospholipid stabilizer layer and can therefore be utilized to probe its structure. Another benefit of the XPPSA method is that not only is the average platelet thickness obtained but also the distribution function [12].

It should be noted that the small-angle scattering patterns of crystalline nanoparticles can also be computed with Debye's equation [13], including the powder average but disregarding the symmetry of the crystalline structure. However, this approach is appropriate only for sufficiently small nanocrystals. Triglyceride suspensions consist of rather large nanocrystals, each of them comprising typically on the order of $10^{7}$ atoms. Applying Debye's equation for ensembles of several hundred of such nanocrystals is computationally too expensive for contemporary computer systems.

For $x$ rays, the scattering length increases linearly with the atomic number $Z$ of the element. Accordingly, the scattering power of organic substances with their light elements is rather low compared to most inorganic compounds. This is different for neutrons where the scattering length increases only with the third root of $Z$ due to potential scattering and is superimposed by a huge erratic component of resonant scattering. Thus, for neutrons large scattering contrasts can be observed between the elements typically present in organic substances. As neutrons interact with the nuclei of the sample, different isotopes of the same element exhibit different scattering lengths. Most prominently, hydrogen $\left({ }^{1} H\right)$ has a much lower coherent scattering length than deuterium $\left({ }^{2} \mathrm{H}\right)$. Protiated molecules possess, therefore, a much higher scattering contrast if they are dispersed in $\mathrm{D}_{2} \mathrm{O}$ instead of $\mathrm{H}_{2} \mathrm{O}$. Furthermore, the scattering contrast of a particular molecule component can be significantly enhanced by a selective deuteration.

In the particular case of lecithins, $\mathrm{x}$ rays are sensitive to the electron-rich phosphate group which facilitates a reliable determination of the phospholipid head group position [14]. Neutron scattering, on the other hand, is not only sensitive to the hydrogen-free phosphate and carbonyl groups in the head group but also to the hydrogen-rich acyl chains that provide a good scattering contrast with respect to the head groups and $\mathrm{D}_{2} \mathrm{O}$. Thus, as a second and complementary method, SANS is a good supplement to SAXS and it will be demonstrated in this contribution that only a combined data evaluation can provide reliable structural information of the stabilizer layer in the studied dispersions. As far as triglyceride suspensions are concerned, SANS was used so far only to prove that the interference maxima of concentrated suspensions at low angles arise from interparticle interferences in the platelet stacks and not from internal structures of the particles [15].

In this contribution, the XPPSA method has been extended to neutron scattering. This made it possible to study the structural arrangement of the phospholipid molecules in the stabilizer layer in more detail. The new simulation method will be used to interpret the SAXS and SANS patterns of dilute $(3 \mathrm{wt} \%)$ tripalmitin suspensions which do not form stacks. They exhibit diffuse particle scattering and the 001 Bragg reflection but lack the complicated superstructure peaks 


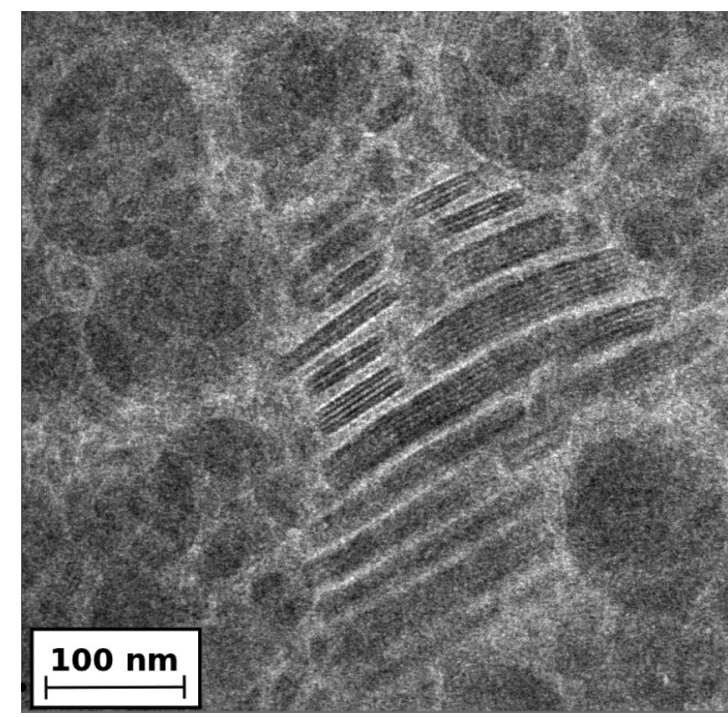

FIG. 3. Cryo-TEM exposure taken from an aqueous tripalmitin suspension stabilized with S100 (10\% tripalmitin, 2.4\% S100, 0.6\% $\mathrm{NaGC}$, in $\mathrm{H}_{2} \mathrm{O}$ ). At tripalmitin concentrations above $4 \%$ a fraction of the particles starts to self-assemble in stacks as shown here [7]. The image reveals the internal structure of the individual platelets that are composed of multiple molecular layers of tripalmitin [5].

that can be observed in concentrated suspensions due to the formation of platelet stacks. In this study, both the pure lecithin DOPC [18:1, Fig. 4(b)] and the lecithin blend Lipoid S100 are used as stabilizers. Lipoid S100 has been used extensively in pharmaceutical studies [1,2,4,6,7,16-18]. Revealing the structure of the adsorbed phospholipids at the surface of the triglyceride nanocrystals is important to understand their stabilization mechanism and polymorphic transformations and to improve their drug encapsulation potential.

In general, the revised XPPSA method presented in this paper is a readily available tool for the study of the adsorption of stabilizer molecules and bioactive molecules like peptides [19], proteins [19-21], and polyelectrolytes like DNA [22] at the solid-liquid interface of solid lipid nanoparticles in future projects.

A direct observation of phospholipids or other molecules in the stabilizer layer with electron microscopy techniques is very difficult. However, cryo-TEM exposures of phospholipid stabilized tristearin and trimyristin suspensions revealed the inner structure of the platelets in their stable $\beta$ modification [5,23]. As shown in Fig. 3 the exposure of a tripalmitin suspension exhibits dark stripes within the platelets that can be attributed to the position of the glycerol backbones of the triglycerides [5]. In some studies [5,23], some platelets exhibit an additional broadening of the outer dark stripes that represent the large (001) surfaces. Due to the electron-rich phosphate group of the phospholipids this was interpreted as an indication of the presence of adsorbed phospholipids on the surface.

\section{MATERIALS AND METHODS}

\section{A. Materials}

Tripalmitin (Dynasan 116, 95\% purity for the fatty acid fraction) was donated by Sasol $\mathrm{GmbH}$, Witten,

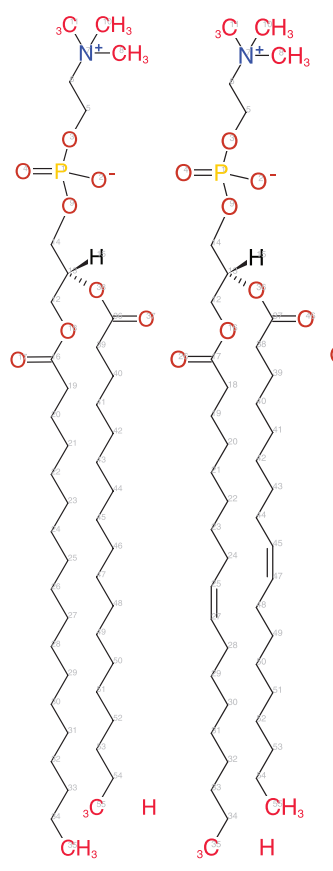

(a) (b)

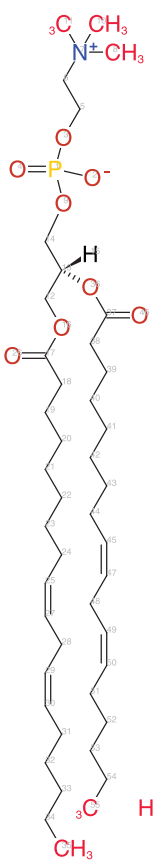

(c)

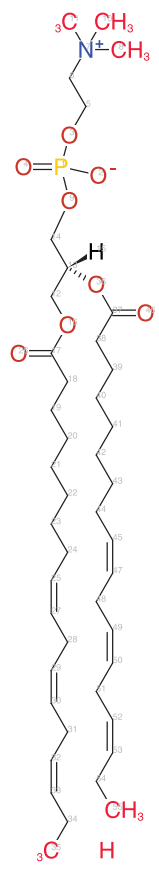

(d)

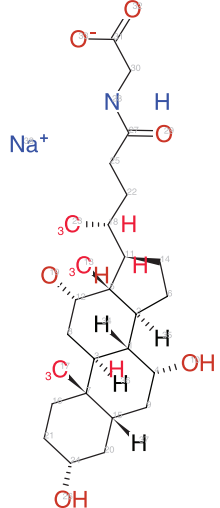

(e)
FIG. 4. (Color online) Chemical structure of phospholipids with different degrees of saturation of the $\mathrm{C}_{18}$ acyl chains in $\mathrm{S100}$. One should keep in mind that $\mathrm{S} 100$ does not consist of solely monoacid phospholipids but also of PC with mixed fatty acids. (a) 18:0 (DSPC), (b) 18:1 (DOPC), (c) 18:2, and (d) 18:3. All phospholipids possess the same head group. For the unsaturated phospholipids the first double bond in the acyl chains is always on the ninth carbon atom as measured from the carboxyl group facilitating a kinked or bent shape of the chains in their natural cis configuration. (e) depicts the structure of the bile salt $\mathrm{NaGC}$ used as a costabilizer.

Germany. Deuterated tripalmitin-d98 was purchased from Dr. Ehrenstorfer GmbH, Augsburg, Germany. S100, a purified soybean lecithin $(\geqslant 94 \%$ phosphatidylcholine (PC), the fatty acid composition according to manufacturers specification is given in Table I), DLPC, and DOPC (purity $\geqslant 98 \%$ ) were provided by Lipoid GmbH, Ludwigshafen, Germany. Chain deuterated DMPC-d54 was purchased from Avanti Polar Lipids Inc., Alabaster, AL, USA. NaGC was purchased from Sigma-Aldrich Chemie GmbH, Taufkirchen, Germany, and $\mathrm{D}_{2} \mathrm{O}$ from Euriso-Top GmbH, Saarbrücken, Germany.

TABLE I. Fatty acid fraction (normalized to the total number of fatty acids) in S100. On average, $86 \%$ of its fatty acids consists of $\mathrm{C}_{18}$ chains; $83 \%$ thereof possess at least one unsaturated bond $(18: x, x>0)$.

\begin{tabular}{lcc}
\hline \hline Fatty acids & & $\%$ \\
\hline Palmitic & $(16: 0)$ & $12-17$ \\
Stearic & $(18: 0)$ & $2-5$ \\
Oleic & $(18: 1)$ & $11-15$ \\
Linoleic & $(18: 2)$ & $59-70$ \\
$\alpha$-linolenic & $(18: 3)$ & $3-7$ \\
\hline
\end{tabular}




\section{B. Sample preparation}

Dilute tripalmitin suspensions were prepared from $3 \%$ tripalmitin, $0.79 \%$ phospholipid (DOPC or S100), and $0.079 \%$ $\mathrm{NaGC}$ in $\mathrm{D}_{2} \mathrm{O}$. For a similar dilute suspension, fully deuterated tripalmitin-d98 and chain deuterated DMPC-d54 was used. All concentrations are given in wt $\%$ for an equivalent fully protiated suspension. Addition of the costabilizer $\mathrm{NaGC}$ is crucial in order to prevent gelation of the nanoemulsions during recrystallization $[1,2]$. Similarly, concentrated suspensions were prepared with $10 \%$ tripalmitin, $2.4 \%$ S100 or DLPC, and $0.6 \% \mathrm{NaGC}_{\text {in }} \mathrm{D}_{2} \mathrm{O}$ (SAXS, SANS) or $\mathrm{H}_{2} \mathrm{O}$ (TEM).

The phospholipid and tripalmitin (melting point $66^{\circ} \mathrm{C}$ ) were heated up to $80^{\circ} \mathrm{C}$ until a homogeneous turbid melt (DOPC, DLCP, DMPC-d54) or a clear yellowish melt (S100) was obtained. $\mathrm{NaGC}$ was dissolved in $\mathrm{D}_{2} \mathrm{O}$ and heated to the same temperature. The mixture was predispersed for $3 \mathrm{~min}$ at $80^{\circ} \mathrm{C}$ with an Ultra-Turrax T25 Basic disperser (IKA-Werke $\mathrm{GmbH} \& \mathrm{Co}$. KG, Staufen, Germany) at $22000 \mathrm{rpm}$. The hot pre-emulsion $(35 \mathrm{ml}$ ) was passed into a continuous APV-2000 high-pressure melt homogenizer (APV Deutschland GmbH, Unna, Germany) which was preheated with an electric heating tape to temperatures of about $65^{\circ} \mathrm{C}$ (m.p. of bulk tripalmitin). The dispersions were homogenized at successively increasing pressure between 1 and $2 \mathrm{kbar}$ for $4 \mathrm{~min}$. With typical flow rates of $2.5-3 \mathrm{ml} / \mathrm{s}$, this corresponds to about $17-21$ cycles. A previous study showed that the particle size does not further decrease after about 16 cycles for this homogenizer and this kind of dispersions [24]. The nanoemulsions were allowed to cool down to room temperature and finally stored at $6^{\circ} \mathrm{C}$.

\section{Small- and wide-angle $x$-ray scattering}

SAXS and wide-angle $\mathrm{X}$-ray scattering (WAXS) exposures were taken on a Kratky-type camera (S3-MICROpix, Hecus XRay Systems GmbH, Graz, Austria) at the FRM II in Garching, Germany. The source is a $50 \mathrm{~W}$ x-ray generator with a copper anode, equipped with microfocusing and monochromatizing biellipsoidal FOX 3D graded W/Si multilayer mirror optics (both from Xenocs, Sassenage, France). The $\mathrm{Cu} K_{\alpha}$ wavelength of $1.5418 \AA$ is selected at a flux of about $3 \times 10^{8} \mathrm{cps}$ (measured with a pin diode) and the primary beam is focused onto the detector. The Kratky block collimator (with 200and $1000 \mu \mathrm{m}$ slits in the vertical and horizontal directions) provides a beam size of about $0.2 \times 0.25 \mathrm{~mm}^{2}$ at the sample position and a flux of about $10^{7} \mathrm{cps}$, as measured with a pin diode. The beam path in the camera housing is completely in vacuum ( 2 mbar) to reduce the air background scattering. Twodimensional SAXS patterns were recorded with a Pilatus $100 \mathrm{~K}$ detector (Dectris AG, Baden, Switzerland). For the detection of the WAXS signal, a Mythen $1 \mathrm{~K}$ line detector (Dectris AG) was used. The suspensions were contained in a home-built capillary sample holder. The quartz capillary (Hilgenberg $\mathrm{GmbH}$, Malsfeld, Germany) had a mean diameter of $1 \mathrm{~mm}$ and a wall thickness of $10 \mu \mathrm{m}$ according to the manufacturer's specification. The sample and the $\mathrm{D}_{2} \mathrm{O}$ background were measured in the same capillary which allows for an accurate background subtraction. The temperature of the sample stage housing the capillary holder was set to $30^{\circ} \mathrm{C}$ for all measurements.

The sample-detector distance is $289.5 \mathrm{~mm}$ and the $2 \theta$ scale of the camera was calibrated using a silver behenate standard (Eastman Kodak Co.) possessing a long spacing of $58.38 \AA$ [25]. Transmission was measured for $0.1 \mathrm{~s}$ with the Pilatus detector and the tungsten beamstop being removed. The ratio of the integrated primary beam intensity with and without the sample in the beam was used to calculate the transmission. The real thickness of the capillary was calculated with the transmission runs for the empty capillary and the capillary filled with $\mathrm{D}_{2} \mathrm{O}$ to be $0.76 \mathrm{~mm}$ [26]. Calibration of the scattering intensity on an absolute scale was done using a glassy carbon sample [27], kindly provided by the 15ID-D USAXS beamline at the Advanced Photon Source, Argonne, IL, USA. Using the calibration factor obtained with the glassy carbon measurement, the transmission for each sample and the thickness of the capillary, each scattering curve can be put on an absolute scale [28]. The data reduction providing the one-dimensional scattering function (azimuthal average, absolute scale) was performed using FIT2DCORR [29], a C++-extension program for FIT2D [30] using LIBTIFF [31]. The scattering pattern of $\mathrm{D}_{2} \mathrm{O}$ was subsequently subtracted from those of the samples. The WAXS data were calibrated with pure tripalmitin powder; reference values for the peak positions were provided by the A2 beamline, HASYLAB at DESY (Hamburg, Germany).

A concentrated tripalmitin suspension with $\mathrm{S} 100$ prepared in $\mathrm{H}_{2} \mathrm{O}$ was measured at the (U)SAXS high-brilliance beamline ID02 at the ESRF in Grenoble [32]. Samples were measured for $0.1 \mathrm{~s}$ at 1.6 and $6 \mathrm{~m}$ sample-detector distances with a wavelength of $\lambda=1 \AA$. For each sample run the transmission was simultaneously obtained. The samples were contained in a homemade multiposition sample holder consisting of silver cells (diameters, $10 \mathrm{~mm}$; sample thicknesses, $1 \mathrm{~mm}$ ) sandwiched between two copper blocks. The cells are covered on both sides by suitable mica windows (about $20 \mu \mathrm{m}$ thick). The sample temperature was equilibrated at $30^{\circ} \mathrm{C}$ using an external water bath. For the absolute intensity calibration $1 \mathrm{~mm}$ water was used as a primary standard. The azimuthally averaged SAXS patterns were obtained with the software package SAXSPROGRAMS [33] and finally the $\mathrm{H}_{2} \mathrm{O}$ background was subtracted from the sample patterns.

\section{Small-angle neutron scattering}

Most of the tripalmitin suspensions were measured at the KWS-2 beamline [34] of FZ-Jülich at the FRM II. The velocity selector provided a wavelength of $\lambda=4.5 \AA$. The wavelength spread was set to $\Delta \lambda / \lambda=20 \%$. The samples were measured at 2,8 , and $20 \mathrm{~m}$ sample-detector distances to access an $s$ range $[s=Q /(2 \pi)]$ of $0.004-0.54 \mathrm{~nm}^{-1}$. The collimation length of the instrument was set to $20 \mathrm{~m}$ followed by an entrance aperture of $30 \times 30 \mathrm{~mm}^{2}$. The sample aperture was chosen to $8 \times 8 \mathrm{~mm}^{2}$. To achieve good statistics the exposures were taken for 10,5 , and $10 \mathrm{~min}$ for the sample detector distances of 2, 8, and $20 \mathrm{~m}$, respectively. Sample transmissions were measured for $2 \mathrm{~min}$ at $8 \mathrm{~m}$ collimation length and sample-detector distance. The samples were contained in Hellma QX 404 quartz cuvettes with $0.5 \mathrm{~mm}$ sample thickness (Hellma GmbH, Müllheim, Germany) placed in a copper-made multiposition sample holder provided at the beamline. The temperature was set for all measurements to $30 \pm 1{ }^{\circ} \mathrm{C}$ with an external water bath. 
Data reduction was done with the software QTIKWS [35]. The two-dimensional raw data were corrected for dark current (measured by placing a boron carbide slab at the sample position), for transmission, and for detector sensitivity (measured with a $1.5 \mathrm{~mm}$ thick sheet of Plexiglas). The Plexiglas runs were also used to put the data on an absolute scale. No $\mathrm{D}_{2} \mathrm{O}$ measurement was subtracted since the $\mathrm{D}_{2} \mathrm{O}$ scattering can be well approximated by a flat background in the small-angle range which is accounted for by a constant in the fitting procedure.

In a similar way, SANS exposures were taken at the D11 beamline [36] at the high-flux reactor at the ILL in Grenoble. A concentrated tripalmitin suspension (10\%) stabilized with $\mathrm{S} 100$ in $\mathrm{D}_{2} \mathrm{O}$ was measured at $25^{\circ} \mathrm{C}$ in a quartz cuvette (0.3 mm pathlength) at a wavelength of $\lambda=4.5 \AA$ and a wavelength spread of $10 \%$. Three different instrumental setups were used: $2 / 8,8 / 28$, and 28/28 m (sample-detector distances/collimation length). Furthermore, a dilute suspension with deuterated tripalmitin was measured at $30{ }^{\circ} \mathrm{C}$ in a $0.5 \mathrm{~mm}$ quartz cuvette at $\lambda=6 \AA$ at $1.2 / 5.5,8 / 13.5$, and $39 / 40.5 \mathrm{~m}$. For all measurements performed at D11 the transmission was measured at $8 / 8 \mathrm{~m}$ and a $1 \mathrm{~mm}$ water sample was used to put the data on an absolute intensity scale. Data reduction was done with the software LAMP [37] provided by the ILL.

\section{E. Cryo- and freeze-fracture transmission electron microscopy (TEM)}

Cryo- and freeze-fracture TEM preparations were performed at the Center for Electron Microscopy of the Jena University Hospital. For cryo-TEM $2 \mu$ l tripalmitin suspension was applied to a copper grid covered by a holey carbon film (1.2/1.3 Quantifoil Micro Tools, Jena, Germany) and excess of liquid was blotted automatically for $2 \mathrm{~s}$ between two strips of filter paper. Subsequently, the samples were rapidly plunged into liquid ethane (cooled to $-180^{\circ} \mathrm{C}$ ) in a cryobox (Carl Zeiss NTS GmbH, Oberkochen, Germany). Excess ethane was removed with a piece of filter paper. The samples were transferred with a cryotransfer unit (Gatan 626-DH, Pleasanton, CA) into the precooled cryoelectron microscope (Philips CM 120, The Netherlands) operated at $120 \mathrm{kV}$ and viewed under low dose conditions. The images were recorded with a 1k CCD camera (FastScan F114, TVIPS, Gauting, Germany). The observation of the internal structure of the triglyceride platelets [5] is difficult since at high magnifications the samples start to degrade quickly (bubbling).

For freeze fracture a small droplet of the tripalmitin suspension is embedded between two copper sandwich profiles. The sandwiches are rapidly frozen in a liquid propane-ethane mixture (1:1) cooled by liquid nitrogen, placed in a freezefracture unit (BAF 400D, BAL-TEC, Liechtenstein), and fractured in a vacuum chamber at $-140^{\circ} \mathrm{C}$ and $10^{-6}$ mbar. The fractured samples are shadowed under an angle of $35^{\circ}$ with platinum/carbon covering the fracture with a $2 \mathrm{~nm}$ layer of Pt/C. Subsequently, 15-20 nm of carbon is deposited from top in order to stabilize the replicas. The replicas were detached from the copper profiles in deionized water, cleaned for $1 \mathrm{~h}$ in a mixture of methanol and chloroform (1:1), fished up on copper grids, and transferred into a transmission electron microscope
(Zeiss CEM 902A, Carl Zeiss AG, Oberkochen, Germany) operated at $80 \mathrm{kV}$.

\section{F. Photon correlation spectroscopy (PCS)}

Particle size (which provides a good estimate for the average platelet diameter) and polydispersity index were determined by applying the cumulant method to the correlation function measured with a photon correlation spectrometer (Brookhaven Instruments Corporation, Holtsville, NY, USA) consisting of a Mini-L 30 compact diode laser $(30 \mathrm{~mW}, 637 \mathrm{~nm})$ and a BI-200 SM goniometer carrying the photomultiplier tube at a scattering angle of $90^{\circ}$. An entrance slit of $100 \mu \mathrm{m}$ and a wavelength filter of $633 \mathrm{~nm}$ were inserted in front of the photomultiplier. A few droplets of the suspension were dispersed without further filtration in Milli-Q water inside a glass cuvette until a count rate of about $100 \mathrm{kcps}$ was obtained. The samples were measured for $2 \mathrm{~min}$ at room temperature $\left(22{ }^{\circ} \mathrm{C}\right)$.

\section{G. Micro differential scanning calorimetry ( $\mu$ DSC)}

$\mu \mathrm{DSC}$ measurements were carried out using a Micro DSC III (Setaram, Caluire-et-Cuire, France). The samples were heated from $5{ }^{\circ} \mathrm{C}$ up to $70^{\circ} \mathrm{C}$ and subsequently cooled back down to $5^{\circ} \mathrm{C}$ at a scan rate of $0.1^{\circ} \mathrm{C} / \mathrm{min}$. About $200 \mathrm{mg}$ of sample was filled into a batch cell. The corresponding fraction of water in the sample was filled in the reference cell.

\section{H. Simulations}

The suspensions are modeled assuming they are composed of different structural elements [12]. These elements can be individual plateletlike nanocrystals, discussed for the dilute suspensions in this contribution, or stacks of such nanocrystals as they exist in concentrated suspensions (cf. Fig. 3), which will be studied in a forthcoming publication [38]. Further elements can be included, e.g., mixed micelles and liposomes. For the coherence volumes of standard SAXS and SANS experiments (cf. Appendix A), the total scattering intensity for a suspension can be approximated very well as the sum over all scattering intensities from the individual elements. This holds for both dilute and concentrated suspensions, since the coherence volume contains not more than one structural element on average [12].

Phospholipid liposomes or mixed micelles of phospholipids with $\mathrm{NaGC}$ and triglyceride might be present in the suspension and contribute to the overall scattering. Liposomes were found in solid triglyceride suspensions [4] and are presumed to exist in phospholipid stabilized emulsions [39]. However, the amount of liposomes in suspensions can be assumed to be far less than in emulsions because of the strong particle surface expansion upon crystallization [40]. Schubert et al. $[20,41]$ did not detect any liposomes or mixed micelles in triglyceride nanosuspensions at all by means of SAXS and TEM. Similar results were obtained with ultracentrifugation and PCS measurements [42]. It was indicated that even high amounts of phospholipid do not lead to a formation of liposomes from the excess phospholipids but rather to a buildup of multiple phospholipid layers in the interface. The same conclusion was drawn from DLS and NMR 


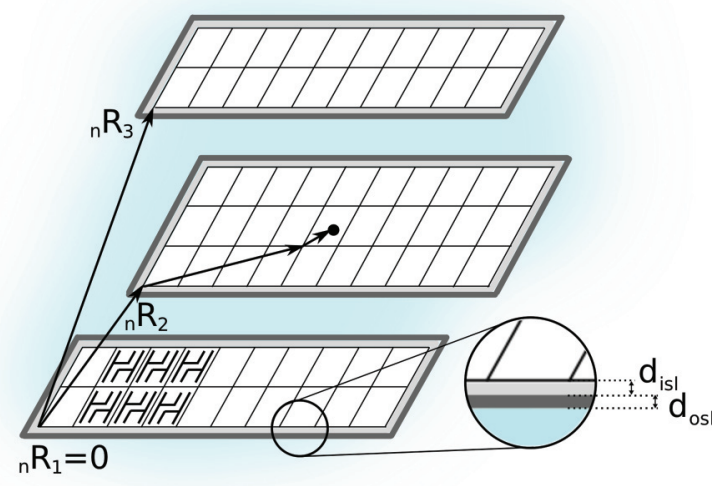

FIG. 5. (Color online) Visualization of the simulation model for an assembly of nanocrystals. For efficient computations the current version of the program allows only the simulation of stacks in which the crystals are parallel to each other. For concentrated triglyceride suspensions this is a good approximation. The vector ${ }_{n} \mathbf{R}_{k}$ points to the $k$ th crystal in the stack. The stabilizer layer consists of two shells dedicated to the phospholipid chains and head groups, respectively. In the lower crystal for some unit cells the tuning fork shape of the tripalmitin molecules in their stable $\beta$ modification (cf. Fig. 10 in Appendix B 2) is indicated.

measurements, indicating phospholipid monolayers for suspensions with lecithin to triglyceride weight ratios below $30 \%$ and supposedly multilayers above that [41].

A monolayer of phospholipids is assumed in the following as the concentration of the lecithin stabilizer is below this threshold for the suspensions studied here. Furthermore, only tripalmitin nanocrystals are considered in the simulations because no liposomes were observed in electron micrographs (cf. Fig. 1).

The model for the structural element of a particle assembly is depicted in Fig. 5. Individual nanocrystals can be considered as a special case of an assembly with only one particle inside. A parallelepipedal shape for the nanocrystals is assumed [12].

The monolayer of phospholipids in the stabilizer layer is modeled by a double shell surrounding the nanoparticles, taking the amphiphilic character of the phospholipid molecules into account. The outer shell contains the head group, including the phosphocholine group, glycerol backbone, and the carbonyl group; the inner shell contains the acyl chains. The same contrasts and molecular volumes are used for the S100 phospholipids as for DOPC (18:1). This is a reasonable approximation since the head groups for the lecithins in $\mathrm{S} 100$ are exactly the same as in DOPC; the fatty acid chains of S100 consist predominantly of $\mathrm{C}_{18}$ chains (about $86 \%$ on average) and about $83 \%$ thereof possess at least one unsaturated bond (cf. Table I and Fig. 4) and are therefore very similar to DOPC, too. The thicknesses of the inner and outer stabilizer layer shells are denoted by $d_{\text {isl }}$ and $d_{\text {osl }}$. Specific molecular models for the arrangement of the phospholipid molecules in the stabilizer layer are discussed in Sec. III.

For the dilute suspensions considered in this study, the SAXS and SANS patterns of five ensembles with 500 platelets each were computed. All platelets in ensemble $i$ had a thickness of $i$ layers of tripalmitin unit cells. The lateral platelet dimensions in the crystallographic $\mathbf{a}_{1}$ and $\mathbf{a}_{2}$ directions were assumed to follow a Gaussian distribution with mean values of 120 and $100 \mathrm{~nm}$, respectively, and a variance of $20 \mathrm{~nm}$ [12]. A linear combination of these ensembles is fitted simultaneously to the experimental SAXS and SANS patterns in the XPPSA method. The fitted linear combination coefficients $c_{i}$ can be interpreted as the volume fractions of the ensembles in the suspension as shown in Appendix D. The fitted contrasts of the shells $\rho_{\text {isl }}^{X}, \rho_{\text {osl }}^{X}$ and $\rho_{\text {isl }}^{n}, \rho_{\text {osl }}^{n}$ and the shell thicknesses $d_{\text {isl }}$ and $d_{\text {osl }}$ can be used to study the molecular arrangement of the phospholipid molecules in the stabilizer layer. Since the shell thicknesses cannot be fitted directly, they were optimized with a parameter scan in steps of $2 \AA$ for $d_{\text {isl }}$ and $d_{\text {osl }}$ in the range $4 \leqslant d_{\text {isl }}+d_{\text {osl }} \leqslant 32 \AA$ with $d_{\text {isl }}, d_{\text {osl }} \geqslant 2 \AA$. For each step in the scan a new simulation has to be run. The simulated SANS patterns were smeared (cf. Appendix E) prior to the fitting procedure.

Appendices B-G provide a detailed description of the simulation method. Particularly, in Appendix B the extension of XPPSA to include neutron scattering and further enhancements (e.g., performance improvements, calculation of the intensities on an absolute scale, improved powder average) that were essential to carry out this study are presented. Furthermore, computational aspects for the numerically expensive simulation method, as well as the fitting of experimental data sets, are discussed there.

Two simple test cases have been considered in Appendix $\mathrm{H}$ to test the correctness of the method. By comparing integrated Bragg reflection intensities as well as zero- $Q$ limits of the scattering intensity for both simulations and analytical calculations, the validity of the new code can be confirmed.

The source code of the simulation program has been released on sourceforge.net [43] under the name XNDIFF.

\section{RESULTS AND DISCUSSION}

The results and discussion section is structured as follows. Some special aspects of the preparation of the deuterated tripalmitin suspensions and their impact for the SANS measurements are discussed in Sec. III A. The results of the PCS, microcalorimetric and WAXS measurements are presented and discussed in Sec. III B. Finally, Sec. III C focuses on the key issue of this paper, the structural characterization of the phospholipid stabilizer layer in the interface between the solid tripalmitin nanocrystals and the dispersion medium by the improved XPPSA method.

\section{A. Sample preparation for SANS}

As mentioned in the Introduction, the 001 Bragg reflection is a key feature of the scattering patterns that is sensitive to the stabilizer layer properties and the thickness distribution of the platelets. Therefore, some peculiarities in the sample preparation have to be taken into account in order to ensure that this Bragg reflection can be observed experimentally.

For the samples considered in this study protiated tripalmitin $\left(\rho^{n}=0.057 \times 10^{-6} \AA^{-2}\right)$ was dispersed in $\mathrm{D}_{2} \mathrm{O}$ since it provides the best neutron scattering contrast for the tripalmitin nanocrystals and a low incoherent background. However, in SANS data the 001 Bragg reflection is actually rather small (cf. Fig. 2). 
With the aim to enhance the scattering signal of the internal crystal structure of the tripalmitin nanocrystals and especially the scattering of the stabilizer layer, contrast variation measurements have been performed (data not shown). For that purpose $\mathrm{D}_{2} \mathrm{O}$ was partly replaced by $\mathrm{H}_{2} \mathrm{O}$ in the dispersion medium. However, this leads to a decrease in the diffuse small-angle scattering and a vanishing $001 \mathrm{Bragg}$ reflection intensity in the incoherent scattering background from the protiated water. Further SANS measurements with spin polarization are planned as this method makes it possible to separate spin-coherent and incoherent scattering [49]. This would make it possible to reduce significantly the incoherent background mainly produced by the light hydrogen in the sample.

Also deuterated tripalmitin-d98 $\left(\rho^{n}=7.96 \times 10^{-6} \AA^{-2}\right)$ does not enhance the coherent scattering for the $001 \mathrm{Bragg}$ reflection. Calculations of the $\mathrm{x}$-ray and neutron structure factors $\left|F_{00 l}\right|^{2}$ for $\beta$-tripalmitin(-d98) with XNDIFF indicated actually a decrease of the 001 Bragg reflection intensity. This was confirmed experimentally with a dilute tripalmitind98 suspension stabilized with the phospholipid DMPC-d54, where the 001 Bragg reflection intensity almost disappears (red curve in Fig. 2) and only a small 002 reflection can be observed. As can be seen in Fig. 14(b) in Appendix H for both, x-ray and neutron scattering, the structure factors $\left|F_{001}\right|^{2}$ and $\left|F_{003}\right|^{2}$ for tripalmitin are bigger than $\left|F_{002}\right|^{2}$ and indeed the $\left|F_{001}\right|^{2}$ structure factor for neutron scattering turns out to be distinctly smaller than the one for x-ray scattering. In contrast, for tripalmitin-d98 the structure factor $\left|F_{002}\right|^{2}$ is bigger than the $\left|F_{001}\right|^{2}$ and $\left|F_{003}\right|^{2}$ values, respectively, which virtually disappear. The structure factors were successfully cross-checked with the program package FULLPROF [50].

\section{B. Sample characterization}

The dilute $(3 \%)$ tripalmitin suspensions stabilized with DOPC and S100, respectively, show a monomodal but polydisperse particle size distribution with diameters ( $z$ average) of 120 and $142 \mathrm{~nm}$ and polydispersity indices of 0.18 and 0.16 , respectively. The corresponding polydispersities $\sigma$ are 21.6 and $22.7 \mathrm{~nm}$ for DOPC and S100, respectively. Particle sizes remained stable over 4 weeks and no macroscopic particles were observed.

The $\mu \mathrm{DSC}$ heating and cooling runs for the suspensions stabilized with DOPC or S100 are displayed in Fig. 6. The first peak at $45^{\circ} \mathrm{C}$ for DOPC and $43{ }^{\circ} \mathrm{C}$ for $\mathrm{S} 100$ in the heating runs has been observed also in other studies of similar suspensions [51]. It has been shown that it is caused by small $(20-30 \mathrm{~nm})$ nanocrystals that crystallize in a phase that has a different WAXS pattern from all the known phases of tripalmitin [51]. WAXS patterns (data not shown) of the suspensions used in this study are dominated by the reflections of the $\beta$ phase but show also two small reflections at $s=2.32$ and $2.44 \mathrm{~nm}^{-1}$ that are linked to the unknown phase [51].

A complicated melting behavior can be observed at higher temperatures which is very similar for both samples. The melting curves with their partially overlapping melting peaks can be interpreted as a sequence of size-dependent melting events of $\beta$-tripalmitin nanocrystals [16,17,51]. Each peak corresponds to an ensemble of platelets with

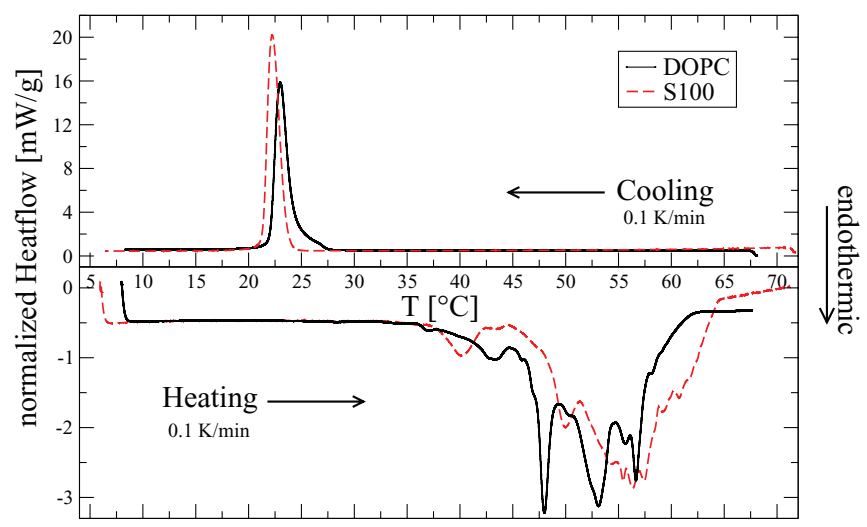

FIG. 6. (Color online) $\mu$ DSC heating and cooling runs for dilute (3\%) tripalmitin suspensions stabilized with the phospholipid DOPC (18:1) and purified soybean lecithin (S100).

a particular thickness. The integrated enthalpies of fusion are 4.79 and $4.81 \mathrm{~J} / \mathrm{g}$ for the suspensions with $\mathrm{S} 100$ and DOPC, respectively. Relative to the mass of tripalmitin in the suspensions this corresponds to 176.3 and $177.5 \mathrm{~J} / \mathrm{g}$, close to the enthalpy of fusion of $188 \mathrm{~J} / \mathrm{g}$ for bulk $\beta$-tripalmitin [52]. The enthalpies of the first melting peak amount to 0.58 and $0.55 \mathrm{~J} / \mathrm{g}$ for S100 and DOPC, respectively, which corresponds to about $12 \%$ of the total enthalpy of fusion for both dispersions. It can, therefore, be concluded that the fraction of the unknown phase is comparatively small in the suspensions and the nanocrystals crystallize mainly in the $\beta$ phase.

The cooling curve of the sample stabilized by $\mathrm{S} 100$ exhibits a sharp crystallization peak at $22.4^{\circ} \mathrm{C}$ with an onset around $25^{\circ} \mathrm{C}$. For the DOPC stabilized sample the crystallization is more complex, starting with a pretransition at $28^{\circ} \mathrm{C}$ and the main transition has its maximum at $22.9^{\circ} \mathrm{C}$. The slightly higher crystallization temperature of the suspension stabilized with DOPC as compared with the one stabilized with S100 is in accordance with previous studies [18], where it was shown that phospholipid chains with a higher degree of unsaturation lead to lower crystallization temperatures. The total crystallization enthalpies are -4.67 and $-4.59 \mathrm{~J} / \mathrm{g}$ for S100 and DOPC, respectively. Relative to the mass of tripalmitin in the suspensions this corresponds to -169.2 and $-171.8 \mathrm{~J} / \mathrm{g}$, again close to the $188 \mathrm{~J} / \mathrm{g}$ for bulk $\beta$-tripalmitin.

The origin of the pretransition for DOPC which amounts to about $-0.57 \mathrm{~J} / \mathrm{g}$ (about $12 \%$ of the total crystallization enthalpy) is unclear. Triglyceride suspensions with fully saturated phospholipids show similar pretransitions in their cooling curves with temperatures roughly related to the transition temperatures of the saturated phospholipid [18]. In contrast, suspensions stabilized with egg yolk or S100 [both being composed of saturated but also a significant or in the case of S100 even dominant fraction of (poly)unsaturated phospholipids] do not show any pretransition. Bunjes et al. argued that the pretransition might be explained by solidification of the saturated chains on the surface of the triglyceride droplets, which also hinders their crystallization into the stable $\beta$ phase that is accompanied by a vigorous surface expansion from spherical droplets to thin platelets. On the other hand, it was argued that the fluid chains of unsaturated phospholipids facilitate the direct transition into the $\beta$ phase (these phospholipids do 
not possess any transition in the relevant temperature range). Whether the observed pretransition for DOPC is related to a solidification of its monounsaturated chains (18:1) is, however, questionable, since for the suspensions with egg yolk lecithin that consists mainly of 18:1 (ca. 30\%), 16:0 (ca. 30\%), and 18:0 (ca. 13\%) chains no such pretransition was observed [18].

For the dispersions studied here the phospholipid was dispersed in the tripalmitin melt instead of dissolving it into the aqueous phase, as was done in previous studies $[2,18]$. For the latter method it was shown that no relevant fraction of the phospholipids forms inverse micelles within the solidified tripalmitin matrix [18]. In order to allow a comparison, dilute tripalmitin suspensions stabilized with the phospholipid DMPC were prepared with both methods. Since for both preparation methods the small-angle scattering patterns, $\mu$ DSC curves, and PCS diameters do not show any significant deviations (data not shown), it was assumed that the phospholipids do not form significant amounts of inverse micelles in the particles using either method.

\section{Molecular models for the stabilizer layer}

First of all, it was tested if the SAXS and SANS patterns of the dilute (3\%) tripalmitin suspensions stabilized with DOPC or S100 (cf. Fig. 7) can be reproduced simultaneously by the same particle model without any particular molecular model

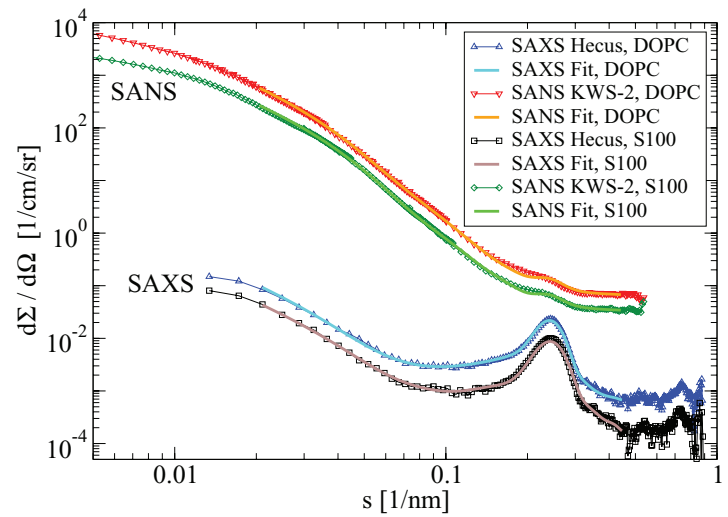

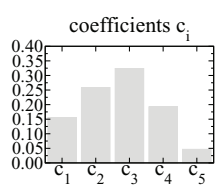

(a)

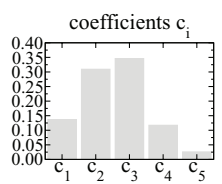

(d)

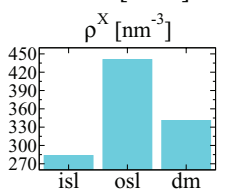

(b)

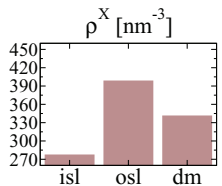

(e)

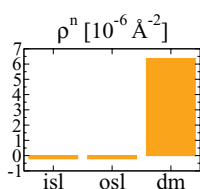

(c)

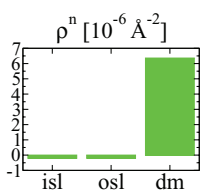

FIG. 7. (Color online) Best simultaneous fits using model A for the SAXS and SANS patterns of dilute (3\%) tripalmitin suspensions stabilized with either DOPC or S100 (for clarity the measured dark green $(\diamond)$, blue $(\triangle)$ and black $(\square)$ curves and their fits are divided by factors 2,5 , and 10, respectively). Panels (a), (b), (c) (for DOPC) and (d), (e), (f) (for S100) display the platelet thickness distribution and the electron and neutron SLDs for the inner and outer stabilizer layers (isl, osl) and the dispersion medium (dm).
TABLE II. Molecular volumes $V$ for DOPC in its liquid crystalline $L_{\alpha}$ phase and water at $30^{\circ} \mathrm{C} \quad[14,44,45]$. Furthermore, the electron densities $\rho^{X}$ and neutron scattering length densities $\rho^{n}$ (neutron coherent scattering lengths taken from [46]) for water and DOPC molecules and its chains and head groups are tabulated. For $\mathrm{NaGC}$ [cf. Fig. 4(e)] the values are estimated in the following way. A crystallographic study for NaGC micellar aggregates (NaGC · 3.375 $\mathrm{H}_{2} \mathrm{O}$ ) revealed a unit cell volume of $V_{u c}=6131 \AA^{3}$ for the tetragonal structure with $Z=8$ [47], leading to a molecular volume of about $6131 / 8-3.375 \times 30=665 \AA^{3}$ for one glycocholate molecule, in good agreement with the $600 \AA^{3}$ found by simple molecular volume considerations [48].

\begin{tabular}{lcccccc}
\hline \hline & Chemical formula & $\begin{array}{c}V \\
\left(\AA^{3}\right)\end{array}$ & $\begin{array}{c}b \\
(\mathrm{fm})\end{array}$ & $\begin{array}{c}\rho^{n} \\
\left(10^{-6} \AA^{-2}\right)\end{array}$ & $\begin{array}{c}\rho^{X} \\
\left(\mathrm{~nm}^{-3}\right)\end{array}$ \\
\hline DOPC & $\mathrm{C}_{44} \mathrm{H}_{84} \mathrm{NO}_{8} \mathrm{P}$ & 1303 & 39.3 & 0.30 & 434 & 333 \\
-Head & $\mathrm{C}_{10} \mathrm{H}_{18} \mathrm{NO}_{8} \mathrm{P}$ & 319 & 60.1 & 1.88 & 164 & 514 \\
-Chains & $\mathrm{C}_{34} \mathrm{H}_{66}$ & 984 & -20.8 & -0.21 & 270 & 274.4 \\
Water & $\mathrm{H}_{2} \mathrm{O}$ & 30 & & & 10 & 332.8 \\
& $\mathrm{D}_{2} \mathrm{O}$ & 29.9 & 19.15 & 6.36 & & \\
NaGC & $\mathrm{C}_{26} \mathrm{H}_{42} \mathrm{NO}_{6}$ & 665 & 59.9 & 0.90 & 253 & 380.5 \\
\hline \hline
\end{tabular}

for the arrangement of the phospholipids in the stabilizer layer (in the following termed as model A). Furthermore, as discussed in Sec. III C, three different molecular models were tested (models B, C, and D). For all models one should bear in mind that the simulation method probes the $\mathrm{X}$-ray and neutron scattering length density profiles of the stabilizer layer shells averaged over the total platelet surfaces. Therefore, no definite conclusions can be drawn on the lateral distribution of the phospholipids.

\section{Model A}

Figure 7 depicts the best simultaneously fitted SAXS and SANS curves obtained with model A for the dilute suspensions stabilized with DOPC and S100, respectively. The corresponding fit parameters are tabulated in Table III and plotted in Figs. 7(a) to 7(f). The shell thicknesses $d_{\text {isl }} / d_{\text {osl }}$ optimized in a parameter scan are $8 \AA / 4 \AA$ for DOPC and $6 \AA / 6 \AA$ for S100 stabilized suspensions. The simultaneous fits provide a good agreement with the data only for thicknesses in the range $10 \leqslant d_{\text {isl }}+d_{\text {osl }} \leqslant 14 \AA$, with $d_{\text {isl }} \geqslant 6 \AA$ and $d_{\text {osl }} \leqslant 6 \AA$. Otherwise, the 001 Bragg reflection in the SANS data cannot be reproduced. When fitting only the SAXS data, many good fits can be found with shell thicknesses typically in the range of $10 \leqslant d_{\text {isl }}+d_{\text {osl }} \leqslant 20 \AA$, with $d_{\text {isl }} \geqslant 6 \AA$ and $4 \leqslant d_{\text {osl }} \leqslant 10 \AA$. Fitting only the SANS data the same limited range of acceptable thicknesses as for the simultaneous fits is found $\left(10 \leqslant d_{\text {isl }}+d_{\text {osl }} \leqslant 14 \AA\right)$. The inclusion of the SANS data makes a more reliable determination of the shell thicknesses possible than the SAXS data alone [12].

For the SANS data the neutron scattering length density (SLD) of the dispersion medium, $\rho_{d m}^{n}$, can be fixed to the SLD of $\mathrm{D}_{2} \mathrm{O}, \rho_{\mathrm{D}_{2} \mathrm{O}}^{n}$ (cf. Table II). However, for the SAXS data it was necessary to allow small variations for the electron density of the dispersion medium, $\rho_{d m}^{X}$, within a few percent above the theoretical value for pure $\mathrm{H}_{2} \mathrm{O}, \rho_{\mathrm{H}_{2} \mathrm{O}}^{X}$ (cf. Table II), in the range $\rho_{d m}^{X} \in[333,345] \mathrm{nm}^{-3}$. Although the samples were prepared 
TABLE III. Parameters for the fits shown in Figs. 7 and 9 with either no molecular model for the stabilizer layer (model A) or model B. The thicknesses of the inner and outer stabilizer layers, $d_{\text {isl }}$ and $d_{\text {osl }}$, the X-ray and neutron scattering contrasts, $\rho^{X}$ and $\rho^{n}$, of the stabilizer layers and the dispersion medium $(\mathrm{dm})$, the scaling factor $\chi$ for the SAXS curve, and the sum over all volume fractions $c_{i}$ are tabulated.

\begin{tabular}{lcccccc}
\hline \hline & & \multicolumn{2}{c}{ No model, A } & & \multicolumn{2}{c}{ Model B } \\
\cline { 3 - 4 } \cline { 6 - 7 } & & DOPC & S100 & & DOPC & S100 \\
\hline$d(\AA)$ & isl & 8 & 6 & & 6 \\
& os1 & 4 & 6 & & 6 \\
$\rho^{X}\left(\mathrm{~nm}^{-3}\right)$ & isl & 283.4 & 277.0 & & 274.0 & 274.0 \\
& osl & 440.6 & 398.2 & & 452.9 & 397.1 \\
& dm & 340.8 & 340.9 & & 340.2 & 341.0 \\
$\rho^{n}\left(10^{-6} \AA^{-2}\right)$ & is1 & -0.21 & -0.21 & & -0.21 & -0.21 \\
& osl & -0.21 & -0.21 & & 3.46 & 4.91 \\
& dm & 6.36 & 6.36 & 6.36 & 6.36 \\
& $\chi$ & 0.938 & 0.870 & & 0.811 & 0.786 \\
& $\sum_{i} c_{i}$ & 0.976 & 0.935 & 1.037 & 1.051 \\
\hline \hline
\end{tabular}

in $\mathrm{D}_{2} \mathrm{O}$, the electron densities for $\mathrm{H}_{2} \mathrm{O}$ can be used since the SAXS scattering function of $\mathrm{H}_{2} \mathrm{O}$ and $\mathrm{D}_{2} \mathrm{O}$ is virtually the same at $30^{\circ} \mathrm{C}$. The electron densities and neutron SLDs for the shells were restricted to the physically meaningful ranges $\rho_{\text {isl }}^{X}, \rho_{\text {osl }}^{X} \in\left[\rho_{c h}^{X}, \rho_{h}^{X}\right]$ and $\rho_{\text {isl }}^{n}, \rho_{\text {osl }}^{n} \in\left[\rho_{c h}^{n}, \rho_{d m}^{n}\right]$. The X-ray and neutron contrasts for the DOPC head group (h) and chains (ch) are tabulated in Table II.

Both $\rho_{\text {isl }}^{X}$ and, in particular, $\rho_{\text {isl }}^{n}$ show a strong preference for protiated acyl chains in the inner shell. As mentioned in the Introduction, $\mathrm{x}$-rays are very sensitive to the electron-rich phosphate group in the head groups. This can indeed be seen in Table III, Figs. 7(b) and 7(e), where $\rho_{\text {osl }}^{X}$ is much higher than $\rho_{d m}^{X}$ and $\rho_{\text {isl }}^{X}$. Nevertheless, the fitted X-ray and neutron contrasts for the outer shell cannot be correlated in a meaningful way. While the fitted electron density for the outer shell clearly favors head groups, the corresponding neutron SLD is much too low and assumes the values for the chains. However, it can be observed that the fitted $\rho_{\text {osl }}^{n}$ are not well defined: While for some good fits the lowest possible SLD is obtained, for others the highest one is chosen.

The distribution of the $c_{i}$ shows a maximum for platelets with a thickness of 3 unit cells, in agreement with a previous study [12].

The obtained thicknesses for the outer shell, 4 and $6 \AA$, are significantly lower than the one obtained in a previous SAXS study with $\mathrm{S} 100$ [12], where $d_{\text {osl }}=13 \AA$ was found. This difference can most probably be attributed to the additional use of SANS data in this study; the SAXS data alone do not determine this parameter well enough. The thicknesses for the inner shell are in good agreement with $d_{\text {isl }}=8 \AA$ found in [12].

The rather small values for $d_{\mathrm{osl}}$ and $d_{\mathrm{isl}}$ indicate a flat arrangement of the head groups in the outer shell and a rather flat arrangement of the chains in the inner shell. The latter can be explained by different approaches.

One explanation might be that the chains lie tilted in the inner shell. Assuming a C-C-C length of $2.54 \AA$ as for alkane chains [53], (saturated) $C_{17}$ chains possess a length of about $22 \AA$. Since $d_{\text {isl }}$ is about $6-8 \AA$, this corresponds to small inclination angles in the range of $16^{\circ}-21^{\circ}$ with respect to the particle surface.

Considering the unsaturated nature of DOPC's and most of S100's fatty acids (all in the cis configuration), the chains might be also kinked. The kinked structure of oleic acid (18:1) gets more bent for linoleic acid (18:2) and assumes a hook shape for $\alpha$-linolenic acid (18:3). For DOPC and the other polyunsaturated fatty acids in S100, the first double bond is at the ninth $\mathrm{C}$ atom counted from the carbonyl group (cf. Fig. 4). This amounts to a shell thickness of about $4 \times 2.54=10.2 \AA$ if the part of the chains from the carbonyl group to the first double bond would stand straight on the surface and the remaining half of the chain would rest on the surface.

Both effects, tilting and a moderate kinking of the fatty acids could also take place simultaneously.

Furthermore, it cannot be fully ruled out that the chains partially stick into the particles when they cosolidify with tripalmitin, leading to seemingly shorter chains.

\section{Models B, C, and D}

Although the fits of model A can reproduce the data very well, confirming the underlying particle model, a molecular model for the phospholipids in the stabilizer layer that is consistent with both SAXS and SANS data has to be derived. Inspired by the results of model A, three molecular models (B, $\mathrm{C}$, and D) are proposed and evaluated in the following.

First, a model for the arrangement of the phospholipids in the stabilizer layer was studied where all head groups are assumed to be situated in the outer shell and all chains in the inner one. This model is referred to as model $\mathrm{B}$ in the following. Figure 8(a) shows a sketch of this model and also indicates that water penetration is allowed for both shells. For a realistic model the levels of water penetration in each shell must be correlated for SAXS and SANS. Furthermore, a volumetric correlation must be applied at the interface of both shells, involving the shell thicknesses and the molecular volumes of the DOPC head group and chains (cf. Table II). As described in detail in Appendix F, this results in three constraints for the X-ray and neutron SLDs that have to be imposed on the fit.

Second, another structural model [model C, Fig. 8(b)] was tested that assumes that all phospholipid molecules reside

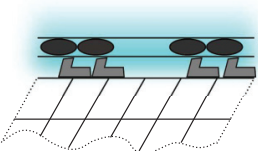

(a)

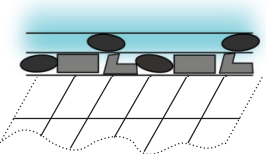

(b)

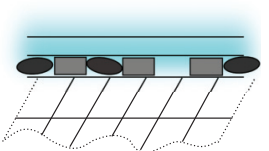

(c)
FIG. 8. (Color online) Models for the molecular arrangement of the phospholipids in the stabilizer layer. (a) Model B: The phospholipid chains of DOPC and $\mathrm{S} 100$ reside in the inner layer, while the head groups stick out in the outer layer. $\mathrm{H}_{2} \mathrm{O} / \mathrm{D}_{2} \mathrm{O}$ penetration is allowed for each layer. (b) Model C: The phospholipid molecules are situated only in the inner shell at the interface; the outer shell is virtually not existent since it is filled with the dispersion medium. (c) Model D: A fraction of the phospholipids resides completely in the inner layer and the remaining fraction occupies both the inner layer with its chains and the outer with the head groups. Water penetration is allowed for the outer layer. 
completely in the inner shell. The outer shell is assumed to be filled with the dispersion medium; water penetration for the inner shell is also included.

Finally, as a kind of hybrid of models B and C, model D [Fig. 8(c)] was tested. As in the case of model C, a fraction of the phospholipid molecules is situated completely in the inner shell (group No. 1) and the remaining fraction is partitioned between both shells as in model B (group No. 2). Similar to model $\mathrm{B}$, three constraints for the scattering contrasts are included in the fit. The outer shell was assumed to consist of the head groups of group No. 2 and water, the inner shell is assumed to contain the complete DOPC molecules of group No. 1 and the chains of group No. 2. The necessary fit constraints are derived in Appendix G.

For all models the fits are computed with dry head groups, i.e., disregarding any interfacial water molecules for the head group. For hydrated DOPC molecules about 11 water molecules enclose the head group, which can be considered as a lower estimate for the bound water of a head group [44]. Using the molecular volume of a water molecule of $30 \AA^{3}$ (cf. Table II), the $\mathrm{x}$-ray and neutron scattering contrasts for the hydrated head group are $\rho_{h}^{X}=422 \mathrm{~nm}^{-3}$ and $\rho_{h}^{n}=4.17 \times$ $10^{-6} \AA^{-2}$, with the hydrated head group volume amounting to $V_{h}+11 \cdot V_{\mathrm{H}_{2} \mathrm{O}}=319+11 \times 30=649 \AA^{3}$. However, models with hydrated head groups do not yield better fits than models with dry head groups (data not shown). This is most probably owed to the fact that water penetration is incorporated independently in the model.

The hydrophilic NaGC costabilizer molecules [cf. Fig. 4(e)] are assumed to arrange themselves flat in the head group region of the phospholipids [54]. A recent quasielastic neutron scattering (QENS) study on the influence of $\mathrm{NaGC}$ on the nanoscopic dynamics of DMPC molecules [55] is in agreement with this assumption. The molar ratio of $\mathrm{NaGC}$ to phospholipids in the suspensions is 1:10 and the molecular volume of a NaGC molecule is approximately twice that of the phospholipid head group (cf. Table II). Assuming that all head groups and NaGC molecules are located in the outer shell, the volume fraction of the NaGC is only about $20 \%$ of the total lipid content in the outer shell. Since the X-ray and neutron SLDs for NaGC are significantly lower than for the phospholipid head group (cf. Table II), NaGC should have only a minor influence on the results for the outer shell and is not considered in the models.

For both suspensions the best simultaneous fits of the SAXS and SANS patterns were obtained with model B (cf. Fig. 9). The fit parameters are tabulated in Tables III \& VI and plotted in Figs. 9(a) to 9(f). For both suspensions the fits show only slightly less agreement with the data than the fits of the unconstrained model A. The optimized shell thicknesses are the same as extracted with model $\mathrm{A}$, indicating again a rather flat arrangement of the phospholipid molecules. The volume fractions of the head groups in the outer shell, $\varphi_{h}$, can be calculated with Eq. (F10) as $64.7 \%$ and $32.4 \%$ for the suspension with DOPC and S100, respectively. The rest of the volume fraction is taken up by the dispersion medium. The volume fraction of the acyl chains in the inner shell $\varphi_{c h}$ is for both suspensions $100 \%$ since the scattering contrasts for the inner shell assume the values of the chains.

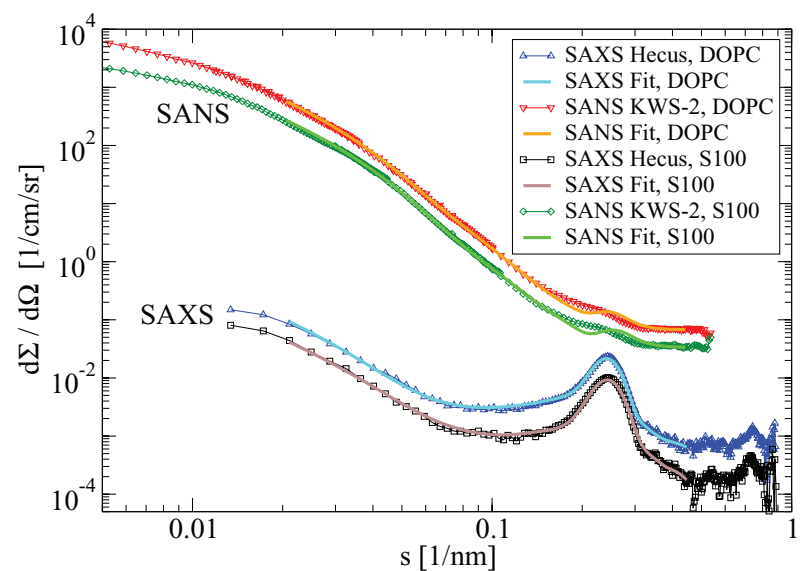

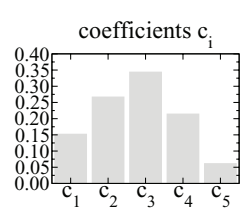

(a)

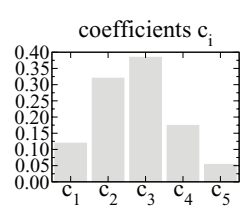

(d)

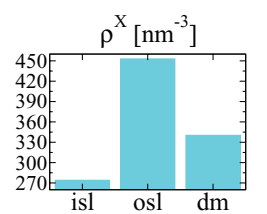

(b)

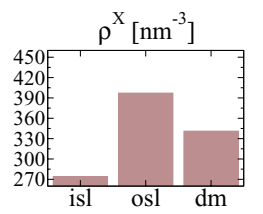

(e)

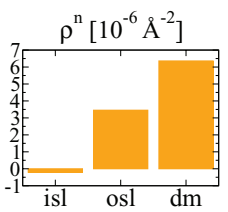

(c)

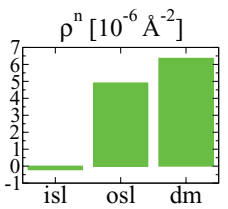

(f)
FIG. 9. (Color online) Best simultaneous fits for the dilute (3\%) tripalmitin suspensions with DOPC or S100 obtained with model B for the stabilizer layer shown in Fig. 8(a). As in Fig. 7, the fitted platelet thickness distributions of the $c_{i}$ and the fitted electron densities and neutron SLDs for the stabilizer shells and the dispersion medium are shown.

Since the inner shell consists only of the acyl chains and water penetration can be observed only for the outer shell, the solid-liquid interface of the nanocrystals seems to be densely covered with the phospholipid molecules. The existence of segregated phospholipid domains at the interface seems therefore improbable.

The fits of model $\mathrm{C}$ are clearly not as good. Omitting the outer shell impairs the fits in particular around the $001 \mathrm{Bragg}$ reflection for both SAXS and SANS. As already observed with models A and B the SAXS data clearly favor a second shell with a higher electron density than the dispersion medium and the inner shell.

Model D provides exactly the same fits as model B. The inner shell comprises only the chains of the phospholipids in group No. 2 but no complete DOPC molecules of group No. 1. For both models, $\mathrm{B}$ and $\mathrm{D}$, it is not favorable to include $\mathrm{D}_{2} \mathrm{O}$ or DOPC into the inner shell since this would increase the electron densities as well as the neutron SLDs of the inner shell. In the limit that only acyl chains are located in the inner shell, models B and D and their constraints in Appendices F and $\mathrm{G}$ become equivalent and, thus, provide the same fit results.

To support the finding that the thickness of the stabilizer layer is significantly lower (12 $\AA$ ) than determined in a previous study ( $21 \AA$ ) [12], an estimate for the total stabilizer layer thickness $\left\langle d_{\mathrm{sl}}\right\rangle$ is derived by volumetric considerations in 
Appendix I. The suspension is assumed to consist of disklike platelets. The sample composition is respected as well as the fitted coefficients $c_{i}$ in Table VI for model B. Table V lists the values for $\left\langle d_{\mathrm{sl}}\right\rangle$ that are for both suspensions on the order of 10 to $11 \AA$. This is in good agreement with the finding of $d_{\text {isl }}+d_{\text {osl }}=12 \AA$ for both suspensions. The slightly larger value here can be explained by the fact that that the outer shell includes also water.

Finally, we would like to point out the ability of the new simulation method to reproduce the SAXS and SANS patterns of crystalline suspensions on an absolute scale with the same particle and stabilizer layer model simultaneously. The fitted scaling factors $\chi$ that regard inaccuracies in the absolute scale for the SAXS curves as well as the sum over all volume fractions $c_{i}$ are close to 1 in Table III for the unconstrained model A. With the more realistic, constrained, model B the deviations are still moderate, e.g., for $\chi$ on the order of $20 \%$.

\section{CONCLUSION}

SAXS and SANS data of dilute DOPC and S100 stabilized tripalmitin suspensions were analyzed simultaneously using a simulation-based data evaluation approach. It was possible to show that the stabilizer molecules form close packed single molecular layers at the interface of the nanoparticles and the dispersion medium. The stabilizer molecules are either assuming an inclined orientation or arrange with their hydrophobic chains not fully stretched. The head group region incorporates water from the dispersion medium. It could clearly be demonstrated that data from both SAXS and SANS are required to draw reliable conclusions on the arrangement of the phospholipid molecules in the stabilizer layer by means of the fitted x-ray densities, neutron contrasts, and shell thicknesses. SANS data are crucial to obtain a reliable estimate for the outer shell thickness. Previous studies which used only SAXS data overestimated the thickness. Estimations of the thickness of the stabilizer layer by volumetric considerations are in good agreement with the total thickness of about $12 \AA$ obtained from the fits. Overall, the SAXS and SANS data of dilute tripalmitin suspensions stabilized with DOPC or S100 could simultaneously be reproduced.

A significantly extended and improved version of the XPPSA method [12] was used for the data evaluation. This method facilitates the simulation of small-angle scattering patterns of crystalline nanosuspensions that contain Bragg reflections in the small-angle range. The simulation method is particularly useful for suspensions of plateletlike nanocrystals that show broad Bragg reflections. It can provide the thickness distribution of the crystals as well as the thicknesses and the scattering contrasts of a stabilizer layer that covers the nanocrystals. The key features include an extension for neutron scattering, a revised powder average algorithm, evaluation of the scattering intensity on an absolute scale, a much higher computing speed, the use of modern HPC clusters, and the opportunity to fit the x-ray and neutron SLDs for the shells and dispersion medium.

This simulation method will now be used for the analysis of concentrated suspensions and other kinds of highly complex nanosuspensions, such as drug loaded suspensions of organic nanoparticles.

\section{ACKNOWLEDGMENTS}

This work was funded by Project No. UN267/2-1 of the Deutsche Forschungsgemeinschaft (DFG) and is based on experiments performed at the Jülich Centre for Neutron Science JCNS, Forschungszentrum Jülich, Germany. We acknowledge the ESRF and the ILL for provision of beam time at the ID02 and D11 beamlines, respectively. Furthermore, we would like to thank the RRZE for using the Woodcrest HPC cluster, R. Gilles and the FRM II for granting SAXS beam time, R. Kaiser and S. Nietzsche from the EMZ Jena for their support with the electron micrography, and $\mathrm{C}$. Bär for providing the capillary holder.

\section{APPENDIX A: COHERENCE VOLUME}

As already mentioned in [12] it is crucial to think about the coherence volume related to SAXS and SANS measurements. Only the scattering of particles or structures arising within the same coherence volume can interfere. To observe, e.g., the peaks arising from inter-particle interferences between the platelets in a stack in concentrated dispersions [6,12], the distance between the particles has to be smaller than the coherence length in the experiments. The knowledge of the experimentally accessible coherence volume makes it possible to decide which kind of structures have to be regarded in the simulations. For small-angle scattering experiments the coherence volume is usually large enough to cover not only individual platelets (with a diameter of about $100 \mathrm{~nm}$ in this study) but also particle stacks as depicted in Fig. 5 with typical interparticle distances of $35 \mathrm{~nm}$.

The coherence volume comprises of the transversal and longitudinal (temporal) coherence lengths. As shown in Eqs. (A1) and (A2) the beam divergence $\Delta \theta$ (determined by the collimation system) and the fractional wavelength bandwidth $\Delta \lambda$ set upper limits on the transversal and longitudinal coherence lengths, respectively [56].

For coherent scattering in the transverse direction, the transverse dimensions in sample $a$ must be smaller than the transversal coherence length $\xi_{t}$. This condition can be expressed with the beam divergence as $[12,56]$

$$
\xi_{t}=\frac{\lambda}{\Delta \theta} \simeq \lambda \frac{L_{c}}{d}>a,
$$

where $L_{c}$ denotes the collimation length of the instrument and $d$ the size of the entrance aperture. For the SANS experiments presented here, $L_{c}$ was $20 \mathrm{~m}$ and $d$ was about $30 \mathrm{~mm}$; the SAXS camera with the present setup has a beam divergence of about $1 \mathrm{mrad}$. The transversal coherence length is therefore (with wavelengths $\lambda=4.5 \AA$ for neutrons and $\lambda=1.54 \AA$ for SAXS) for both experiments on the order of a few $100 \mathrm{~nm}$.

For the longitudinal coherence length $\xi_{l}$ it can be shown [56] that for Bragg scattering ( $a$ is a lateral width in the sample) in the small-angle range $(s \lambda \ll 1)$ it must hold

$$
\xi_{l}=\frac{\lambda^{2}}{\Delta \lambda}>a(s \lambda) \sqrt{1-\frac{(s \lambda)^{2}}{4}} \approx a(s \lambda),
$$

making the condition on the longitudinal coherence length dependent on $s$, i.e., the considered length scale. A similar expression can be obtained with $a$ being a characteristic 
thickness in the sample, but since the expression is of the order of $(s \lambda)^{2}$, Eq. (A2) is often more limiting [56].

For SAXS $\Delta \lambda / \lambda$ is usually better than $10^{-2}$ for both benchtop and synchrotron beamlines. With crystal monochromators like $\mathrm{Si}$-(111) or $\mathrm{Ge}-(111)$ often used at synchrotron beamlines, $\Delta \lambda / \lambda$ is about $1.3 \times 10^{-4}$ [57]. For SANS, however, $\Delta \lambda / \lambda$ is usually in the range of $0.05-0.2$. The experiments presented here have $\lambda / \Delta \lambda \gtrsim 5$ for SANS and $\lambda / \Delta \lambda \gtrsim 10^{2}$ for SAXS. The inequality in Eq. (A2) is therefore well fulfilled in the small-angle range and the length scales considered here.

\section{APPENDIX B: SIMULATION METHOD}

In the following the different structural elements in the suspension are classified and designated by an index $i$. For ensembles of individual particles, all nanocrystals with a thickness of $i$ triglyceride unit cells are a member of class $i$, in the following also referred to as ensemble $i$. For particle stacks, one can similarly classify the stacks with an index $i$ identifying the number of nanocrystals in the stack. In the next section we consider only one ensemble and, thus, omit the index $i$.

\section{Extension on neutron scattering}

As shown in [12], based on the kinematic scattering theory, the differential scattering cross section of a particle stack including ${ }_{n} M$ particles can be written as

$$
{ }_{n}\left(\frac{d \sigma}{d \Omega}\right)(\mathbf{Q})=\left|\sum_{k=1}^{n M}\left[e^{i \mathbf{Q} \cdot{ }_{n} \mathbf{R}_{k}}\left({ }_{n} F_{k n} G_{k}-{ }_{n} S_{k}\right)\right]\right|^{2},
$$

where $n$ denotes the $n$th member in the ensemble and $k$ labels the crystals in the stack. just as in single crystal or powder diffraction ${ }_{n} F_{k}$ denotes the structure amplitude that accounts for the scattering contributions from one unit cell and ${ }_{n} G_{k}$ denotes the lattice factor of the $k$ th crystal. ${ }_{n} S_{k}$ describes the shape of the $k$ th particle and includes the contributions from the stabilizer layer shells. The functions ${ }_{n} S_{k}$ can be identified with the particle form factor $P(Q)$ in simulations of homogeneous nanoparticles. The exponential function characterizes the interparticle interferences within the stack. The vectors ${ }_{n} \mathbf{R}_{k}$ from a common origin to the particles are drawn in Fig. 5.

The structure amplitude for the $k$ th crystal is given by

$$
{ }_{n} F_{k}(\mathbf{Q})=\sum_{l=1}^{N_{u c}}\left\{e^{i \mathbf{Q}_{n} \mathbf{r}_{k, l}} \int_{V_{A}^{l}} \rho_{l}(\tilde{\mathbf{R}}) e^{i \tilde{\mathbf{R}} \cdot \mathbf{Q}} d^{3} \tilde{\mathbf{R}}\right\},
$$

where ${ }_{n} \mathbf{r}_{k, l}$ denotes the coordinates of the $l$ th atom (SAXS) or nucleus (SANS) in the unit cell, given in units of the three lattice vectors ${ }_{n} \mathbf{a}_{k, \mu}(\mu=1,2,3)$ of the crystal. $N_{u c}$ denotes the number of atoms in the unit cell and $V_{A}^{l}$ the volume of the $l$ th atom and nucleus, respectively. The spatial distribution of the $\mathrm{X}$-ray and neutron scattering SLDs is given as

$$
\rho_{l}(\mathbf{r})= \begin{cases}r_{e} \rho_{l}^{e}(\mathbf{r}) & \text { x rays, } \\ \frac{b_{l}}{V_{A}^{l}} \delta(\mathbf{r}) & \text { neutrons, }\end{cases}
$$

where $r_{e}=2.8179 \mathrm{fm}$ denotes the classical electron radius (Thomson scattering length), $\rho_{l}^{e}(\mathbf{r})$ the electron density distribution of the $l$ th atom, and $b_{l}$ the bound coherent scattering length of the $l$ th nucleus in the unit cell. Accordingly, the Fourier transform of $\rho_{l}$ in Eq. (B2) can be rewritten as

$$
\mathcal{F}\left\{\rho_{l}(\mathbf{r})\right\}= \begin{cases}r_{e} f_{l}^{0}(Q) & \text { x rays, } \\ b_{l} & \text { neutrons },\end{cases}
$$

where $f_{l}^{0}(Q)$ is the atomic scattering factor for each atom. This factor is computed with the Cromer-Mann coefficients $[58,59]$ that are available from the DABAX [60]. The coherent scattering lengths of the nuclei can be found in the tables of [46] and are provided by the National Institute of Standards and Technology [61].

The lattice factor can be expressed as [12]

$$
{ }_{n} G_{k}(\mathbf{Q})=\prod_{\mu=1}^{3} \frac{\exp \left(i_{n} N_{k, \mu} \mathbf{Q} \cdot{ }_{n} \mathbf{a}_{k, \mu}\right)-1}{\exp \left(i \mathbf{Q} \cdot{ }_{n} \mathbf{a}_{k, \mu}\right)-1},
$$

where ${ }_{n} N_{k, \mu}$ denotes the number of unit cells in the crystallographic $\mu$ direction of the $k$ th particle of the $n$th ensemble member. Finally, ${ }_{n} S_{k}$ is given by the sum

$$
{ }_{n} S_{k}(\mathbf{Q})=\sum_{j=1}^{3} \Delta \rho_{j} \cdot{ }_{n} P_{k, j}(\mathbf{Q})
$$

where the three functions ${ }_{n} P_{k, j}(j=1,2,3)$ are defined as

$$
{ }_{n} P_{k, j}(\mathbf{Q})={ }_{n} V_{k, j} \prod_{\mu=1}^{3} \frac{\exp \left(i_{n} N_{k, \mu} \mathbf{Q} \cdot{ }_{n} \mathbf{a}_{k, \mu, j}\right)-1}{i_{n} N_{k, \mu} \mathbf{Q} \cdot{ }_{n} \mathbf{a}_{k, \mu, j}}
$$

where ${ }_{n} V_{k, 1}={ }_{n} V_{k}$ is the volume of the $k$ th crystal of the $n$th member in the ensemble and ${ }_{n} V_{k, 2}$ as well as ${ }_{n} V_{k, 3}$ additionally include the volume of the inner and inner plus outer stabilizer layer, respectively. Stretched lattice vectors ${ }_{n} \mathbf{a}_{k, \mu, j}$ can be introduced (with ${ }_{n} \mathbf{a}_{k, \mu, 1}={ }_{n} \mathbf{a}_{k, \mu}$ ) that span the enlarged volumes ${ }_{n} V_{k, j}$,

$$
{ }_{n} V_{k, j}=\operatorname{det}\left({ }_{n} \mathbf{a}_{k, 1, j},{ }_{n} \mathbf{a}_{k, 2, j},{ }_{n} \mathbf{a}_{k, 3, j}\right) \prod_{\mu=1}^{3}{ }_{n} N_{k, \mu} .
$$

The scattering contrasts $\Delta \rho_{j}$ in Eq. (B6) are given by

$$
\Delta \rho_{1}=\rho_{\text {isl }}, \quad \Delta \rho_{2}=\rho_{\text {osl }}-\rho_{\text {isl }}, \quad \Delta \rho_{3}=\rho_{d m}-\rho_{\text {osl }},
$$

where $\rho_{\text {isl }}, \rho_{\text {osl }}$, and $\rho_{d m}$ are the X-ray or neutron SLDs of the inner and outer stabilizer layer shell and the dispersion medium, respectively. For x-ray scattering in the following the electron densities will be used, the corresponding x-ray SLD in Eq. (B9) can be obtained by multiplication of the electron densities with $r_{e}$.

Since the particles in the suspension and even in a specific ensemble are not necessarily identical, an ensemble average has to be computed for each ensemble. Typically for each ensemble an average of particles whose diameters follow a Gaussian distribution function with parameters $\mu_{D}$ and $\sigma_{D}$ as in Eq. (I1) has to be taken. For stacks, the interparticle distances (Gaussian distribution with mean distance $\mu_{R}$ and variance $\sigma_{R}$ used in $[12,38]$ ) and the thickness of the particles in the stack will be statistically distributed as well. Furthermore, since the suspension of the nanocrystals can be considered as a powder, the direction-dependent scattering cross section in Eq. (B1) has to be averaged over all relevant orientations in order to obtain the one-dimensional scattering function that 
is also obtained from azimuthal averaging the experimental data of isotropically scattering samples. The orientational and ensemble averaged scattering cross section for a specific ensemble can be calculated as

$$
\left(\frac{d \sigma}{d \Omega}\right)(Q)=\left\langle\left\langle n\left(\frac{d \sigma}{d \Omega}\right)(\mathbf{Q})\right\rangle_{\Omega_{\mathbf{Q}}}\right\rangle_{M},
$$

where $\langle\cdot\rangle_{\Omega_{\mathrm{Q}}}$ indicates the orientational average and $\langle\cdot\rangle_{M}$ the ensemble average over $M$ members in the ensemble. The numerical implementation of the orientational average is an important point for the validity and performance of the simulation method and will be considered in more detail in Appendix B 2. It should be noted that the nanocrystals or the stacks are actually spatially fixed in the simulations and the scattering vector $\mathbf{Q}$ is rotated, which is equivalent to a spatially fixed direction of the $\mathbf{Q}$ vector and a rotation of the particles. The latter interpretation corresponds experimentally to a setup with the scattered intensity of a powder sample or a suspension of nanocrystals recorded with a line detector. For sufficiently low concentrations (typically $\lesssim 15 \mathrm{wt} \%$ ) the scattering patterns of triglyceride suspensions are isotropic [15]. In this study the two-dimensional exposures taken with contemporary SAXS and SANS detectors are reduced by an azimuthal average to the one-dimensional scattering function that corresponds to the simulated scattering patterns with a line detector. However, for higher concentrations the two-dimensional scattering patterns become anisotropic [15]. The present simulation method is not intended to handle these systems, although the integration of orientational distribution functions with up to three Eulerian angles would allow the generation of two-dimensional scattering patterns. For homogeneous (i.e., noncrystalline) nanoparticles, simulations of this type are already described in the literature [62].

\section{Further enhancements of the simulation method}

A severe limitation of the previous simulation method [12] was its low speed. This owed to the fact that the structure amplitude in Eq. (B2) is computed for each particle and orientation which includes a summation over all scattering lengths of the atoms and nuclei in the unit cell, respectively. Typical simulation runs for a fixed parameter set for the scattering contrasts and shell thicknesses with a sufficient fine sampling for the powder average took on the order of several weeks for a single core CPU, making parameter scans effectively impossible. To remedy this restriction, the simulation method was changed in several points.

Since the structure amplitudes ${ }_{n} F_{k}(\mathbf{Q})$ for $\mathrm{x}$-ray and neutron scattering are independent of the ensemble average for individual platelets, the computation of the complex valued ${ }_{n} F_{k}(\mathbf{Q})$, taking about $90 \%$ of the overall computation time, can be done only once, stored in memory, and later reused for all nanocrystals. For a rather fine sampling of the orientations and the modulus of the scattering vector, $Q$, as used in this study, the total memory consumption is on the order of only a few gigabytes. The same strategy can be applied for particle assemblies if the restriction that the platelets are aligned in parallel is acceptable, which is often the case. For suspensions with particle stacks as they have been observed in concentrated triglyceride suspensions [7] and will be considered in an upcoming paper [38], the following trick significantly reduces the computational efforts: When computing, for example, the scattering patterns for an ensemble of particle stacks where each member consists of five particles, the scattering patterns of ensembles of particle stacks containing four, three, or two particles can be obtained at the same time since they are computed when building the stacks step by step. Similarly, the scattering patterns for ensembles of individual platelets with distinct thicknesses are obtained at the same time. To summarize, these improvements increase the speed of the program typically by factors of 10-30 depending on the simulated structural elements.

Nevertheless, even with this considerable performance increase, complete parameter scans, for example for the scattering contrasts (up to six parameters for a SAXS and SANS curve) and thicknesses of the stabilizer layer shells (two parameters), are virtually impossible.

\section{a. Separation of the geometry and scattering contrasts}

Another key feature of the new simulation method is that it is possible to avoid expensive parameter scans for the contrasts of the shells and the dispersion medium. With a given particle model (i.e., with a given geometry) the simulation has to be run only once and the $\mathrm{x}$-ray and neutron scattering contrasts can be later fitted later. Thus, parameter scans are now required only for parameters related to the geometry, e.g., the thicknesses of the shells, mean interparticle distances in stacks, etc. In a straightforward calculation one can show that Eq. (B10) can be expressed as a quadratic form in the scattering contrasts as

$$
\left(\frac{d \sigma}{d \Omega}\right)(s)=\sum_{j=1}^{10} \Gamma_{j} \Upsilon_{j}(s),
$$

with ten solely geometry dependent functions $\Upsilon_{j}$ that are introduced in Eq. (B18) $[s=Q /(2 \pi)]$. The $\Gamma_{j}$ defined as

$$
\Gamma_{j}= \begin{cases}\Delta \rho_{j}^{2}, & j=1,2,3, \\ \Delta \rho_{j_{1}} \Delta \rho_{j_{2}}, & j=4,5,6, \\ \Delta \rho_{j-6}, & j=7,8,9, \\ 1, & j=10,\end{cases}
$$

include the contrasts $\Delta \rho$ from Eq. (B9). $\left(j_{1}, j_{2}\right)$ equals $(3,2)$ for $j=4 ;(3,1)$ for $j=5$ and $(2,1)$ for $j=6$.

To further increase performance the code was parallelized. Parameter scans can now efficiently be performed on high performance computer clusters as it will be described in Appendix B 3.

Up to now only a single ensemble has been considered. Respecting that the suspensions typically consist of different structural elements (e.g., ensembles with different platelet thicknesses or stacks with different numbers of platelets within a stack), in the following the functions $\Upsilon_{j}$ will be indexed additionally with the ensemble index $i$. Generally, this may affect also the functions at lower levels, e.g., the functions ${ }_{n} \xi_{j}$, ${ }_{n} F_{k},{ }_{n} G_{k}$, and ${ }_{n} P_{k, j}$, where the ensemble index $i$ has been suppressed for a better readability.

The same holds for an index $v$ enumerating different simulation runs that might be necessary for different experimental datasets (different samples or scattering techniques) used in the simultaneous fitting routine discussed later in Appendix B 4. 
In the following the index $v$ will be suppressed and only explicitly written in Appendices B 4 and B 5. Nevertheless, one should bear in mind that also the macroscopic cross sections $(d \Sigma / d \Omega)$, and as aforementioned the functions $Y_{j}^{i}, \Upsilon_{j}^{i}$, etc., and, furthermore, also the contrasts $\Gamma_{j}$ and $\Delta \rho_{j}$ in Eqs. (B12) and (B9) carry implicitly the index $v$.

\section{b. Calculation of the intensities on an absolute scale}

Another key feature of the new simulation method is its capability to compute the scattered intensities on an absolute scale; i.e., it provides the macroscopic scattering cross sections $(d \Sigma / d \Omega)$ in units of in $(1 / \mathrm{cm})$, which facilitates a direct comparison with the experimental data and would ideally make any scaling factors redundant. The macroscopic scattering cross section for the $i$ th ensemble can be obtained from Eq. (B11) as

$$
\left(\frac{d \Sigma}{d \Omega}\right)^{i}(s)=\sum_{j=1}^{10} \Gamma_{j} Y_{j}^{i}(s)
$$

where the functions $Y_{j}^{i}$ are related with the $\Upsilon_{j}^{i}$ via

$$
Y_{j}^{i}(s)=\frac{\varphi_{\text {cry }}}{V^{i}} \Upsilon_{j}^{i}(s), \quad j=1, \ldots, 10,
$$

by a normalization with the total simulated dispersion volume for the $i$ th ensemble, which is given by $V^{i} / \varphi_{\text {cry }}$, where $V^{i}$ is the sum over all crystal volumes in the ensemble,

$$
V^{i}=\sum_{n=1}^{M^{i}} \sum_{k=1}^{{ }^{M^{i}}}{ }_{n} V_{k}
$$

and $\varphi_{\text {cry }}$ the volume fraction of the crystals (excluding the shells) in the suspension.

Finally, to obtain the macroscopic scattering cross section of the suspension including different structural elements similarly as in [12] a linear combination,

$$
\left(\frac{d \Sigma}{d \Omega}\right)(s)=\sum_{i} c_{i}\left(\frac{d \Sigma}{d \Omega}\right)^{i}(s),
$$

of the scattering contributions of all considered ensembles has to be fitted to the experimental data. As shown in Appendix D the linear coefficients $c_{i}$ can be interpreted as the volume fractions of ensemble $i$ and Eq. (D7) imposes a completeness relation as a constraint for the $c_{i}$.

The electron densities and/or neutron SLDs that are implicitly contained in the $\Gamma_{j}$ can be obtained by the fitting of Eq. (B16) with Eqs. (B13), (B12), and (B9).

\section{c. Input and transformation of crystallographic structure data}

The unit cell parameters $a, b, c, \alpha, \beta$, and $\gamma$, as well as the atomic positions within the unit cell, can be imported from files using the crystallographic information format (cif). The atomic positions of an entire unit cell can-either automatically with the space group symbol or by user defined symmetry operations - be derived from the asymmetric unit cell given in the cif files with an installed CCP4 library [63] using its pdbset routine or by providing solely the CCP4 syminfo.lib file [64].

For an accurate characterization of the phospholipid stabilizer layer it is crucial to provide an appropriate unit cell with

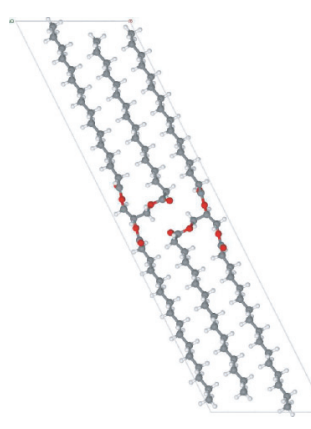

\begin{tabular}{|c|r|r|}
\hline & ref. [65] & this work \\
\hline$a[\AA]$ & 5.4514 & 5.4514 \\
\hline$b[\AA]$ & 11.9450 & 11.9450 \\
\hline$c[\AA]$ & 40.4820 & 46.8425 \\
\hline$\alpha\left[^{\circ}\right]$ & 84.66 & 118.10 \\
\hline$\beta\left[^{\circ}\right]$ & 86.97 & 106.12 \\
\hline$\gamma\left[^{\circ}\right]$ & 79.77 & 79.77 \\
\hline$V_{u c}\left[\AA^{3}\right]$ & 2581 & 2581 \\
\hline$d_{001}[\AA]$ & 40.21 & 40.21 \\
\hline
\end{tabular}

FIG. 10. (Color online) Visualization of the transformed triclinic unit cell of tripalmitin's stable $\beta$ phase used in the simulations (view to the $b-c$ plane). The structural data of $\beta$ tripalmitin from Ref. [65] are summarized in the table as well as the data for the transformed unit cell. The unit cell contains $Z=2$ tripalmitin molecules that adopt a characteristic tuning fork structure in the $\beta$-phase [66].

full tripalmitin molecules inside that do not overlap with the surrounding stabilizer layer. Since the unit cell and the fractional coordinates from the cif file from Ref. [65] together with the default symmetry operations for the fractional coordinates of the triclinic space group P-1 $(x, y, z$ and $-x,-y,-z)$ do not meet this condition a transformed unit cell has been used (cf. table in Fig. 10). The new unit cell (lattice vectors $\mathbf{a}_{i}$ ) is related to the cell from Ref. [65] (lattice vectors $\tilde{\mathbf{a}}_{i}$ ) by the transformation

$$
\mathbf{a}_{i}=\sum_{j} T_{i j} \tilde{\mathbf{a}}_{j}, \quad \text { where } \quad T=\left(\begin{array}{ccc}
1 & 0 & 0 \\
0 & 1 & 0 \\
-2 & -2 & 1
\end{array}\right) .
$$

The fractional coordinates in the new unit cell $\xi_{1}, \xi_{2}, \xi_{3}$ derive from the fractional coordinates $\tilde{\xi}_{1}, \tilde{\xi}_{2}, \tilde{\xi}_{3}$ of the cif file from Ref. [65] via the transformations $\xi_{1}=\tilde{\xi}_{1}+2 \tilde{\xi}_{3}, \xi_{2}=\tilde{\xi}_{2}+2 \tilde{\xi}_{3}$, $\xi_{3}=\tilde{\xi}_{3}$ and the symmetry operations $x, y, z$ and $1-x, 1-$ $y, 1-z$ must be applied for the $\xi_{i}$. By this a transformed unit cell as shown in Fig. 10 is obtained.

\section{d. Powder and ensemble average}

A crucial point for the simulation of dispersed nanocrystals is the quality of the spherical code [67] used for the powder average. In the earlier program version used in [12] a so-called grid algorithm was used that covers the virtual unit sphere $\mathbb{S}^{2}$ representing all possible orientations of a nanocrystal with a grid of lines of longitude and latitude (lines of constant azimuthal angles $\varphi$ and polar angles $\vartheta$, respectively). The points of intersection of these grid lines represent the orientations that are sampled in the powder average.

To address the question of the validity and performance of the grid algorithm, an additional spherical code that distributes the orientations of the nanocrystals randomly on the sphere was implemented as a reference (Monte Carlo algorithm). Generally, the ensemble average over all $M$ members (individual particles or particle stacks) in the ensemble and the powder average for each ensemble member can be written as

$$
\Upsilon_{j}(s)=\sum_{n=1}^{M}\left(\frac{\sum_{v=1}^{N_{\vartheta}} \sum_{u=1}^{N_{\varphi}} w_{u v} \cdot{ }_{n} \xi_{j}\left(\mathbf{Q}_{u v}\right)}{\sum_{v=1}^{N_{\vartheta}} \sum_{u=1}^{N_{\varphi}} w_{u v}}\right),
$$


when using a spherical code with up to two independent variables $\varphi$ and $\vartheta$. Hereby, the functions ${ }_{n} \xi_{j}$ are given by Eqs. (C1) to (C4) for particle stacks and Eq. (C5) for individual particles in Appendix $\mathrm{C}$, involving each the structure amplitudes ${ }_{n} F_{k}$, the lattice amplitudes ${ }_{n} G_{k}$, and the shell related functions ${ }_{n} P_{k, j}$. For a grid algorithm, $N_{\varphi}$ and $N_{\vartheta}$ denote the numbers of sample points for the angular variables $\varphi$ and $\vartheta$ spanning the grid over $\mathbb{S}^{2}$. For the grid method $\mathbf{Q}_{u v}$ is defined as

$$
\mathbf{Q}_{u v}=2 \pi \cdot s \cdot\left(\cos \varphi_{u} \sin \vartheta_{v}, \sin \varphi_{u} \sin \vartheta_{v}, \cos \vartheta_{v}\right)^{T}
$$

in the basis of three orthonormal vectors in reciprocal space. Thereby $\varphi_{u}$ and $\vartheta_{v}$ denote an azimuthal and polar angle, respectively. For this study the third basis vector was chosen as the reciprocal lattice vector $\mathbf{G}_{001}$.

Eventually, the $w_{u v}$ in Eq. (B18) describes the weights that apply for a spherical code in order to distribute the points uniformly over the unit sphere $\mathbb{S}^{2}$. The weights that need to be applied for the grid algorithm are discussed at the end of this section. For a Monte Carlo algorithm distributing $N$ points randomly on $\mathbb{S}^{2}$ and for which no weights have to be applied, i.e., $w_{u v}=1, \forall u, v$ and $\sum_{u v} w_{u v}=N$, the $\Upsilon_{j}(s)$ in Eq. (B18) can be rewritten as

$$
\Upsilon_{j}(s)=\sum_{n=1}^{M}\left(\frac{\sum_{v=1}^{N} n \xi_{j}\left(\mathbf{Q}_{v}\right)}{N}\right),
$$

with the $\mathbf{Q}_{v}$ being randomly chosen by an appropriate algorithm.

For centrosymmetric triclinic crystals like tripalmitin it is sufficient to sample only the upper half of $\mathbb{S}^{2}$, i.e., the angular ranges $\varphi=[0,2 \pi)$ and $\vartheta=[0, \pi / 2)$, for any spherical code.

Different methods for the implementation of Monte Carlo codes that distribute points randomly on the (upper) $\mathbb{S}^{2}$ in such a way that the surface is homogeneously covered do exist [68]. The first method basing on simple coordinate transformations ensures that each differential element of solid angle, $d \Omega_{\mathbf{Q}}=\sin \vartheta d \vartheta d \varphi=-d(\cos \vartheta) d \varphi$ will include the same number of points on the sphere. Using independent random variables $u, v \sim U_{(0,1)}$ being uniformly distributed in $(0,1)$, the azimuthal and polar angles can be obtained by the coordinate transformations $\varphi_{u}=2 \pi \cdot u$ and $\vartheta_{v}=\arccos v$, respectively.

Another Monte Carlo code implemented uses Marsaglia's method [69]. Both methods give quantitatively very similar results, and, thus, the first method was chosen because of better performance. For both methods a C++ library [70] of a Mersenne Twister pseudorandom number generator [71] was used to generate the numbers $u$ and $v$ in XNDIFF.

Figure 11 displays the SAXS patterns calculated for 100 individual tripalmitin platelets with statistically varying diameters and thicknesses that are powder and ensemble averaged using different spherical codes and different numbers of orientations. The diffractograms obtained with the existing implementation of the grid algorithm (brown and black dashed curve) do not match the simulations using the Monte Carlo algorithm [eight simulations, each with 4320 points (blue curves), and three simulations, each with 64800 points (cyan curves)].

The cause of this discrepancy is the suppression, discussed in [12], of the $\sin \vartheta$ weighting factor used typically in grid

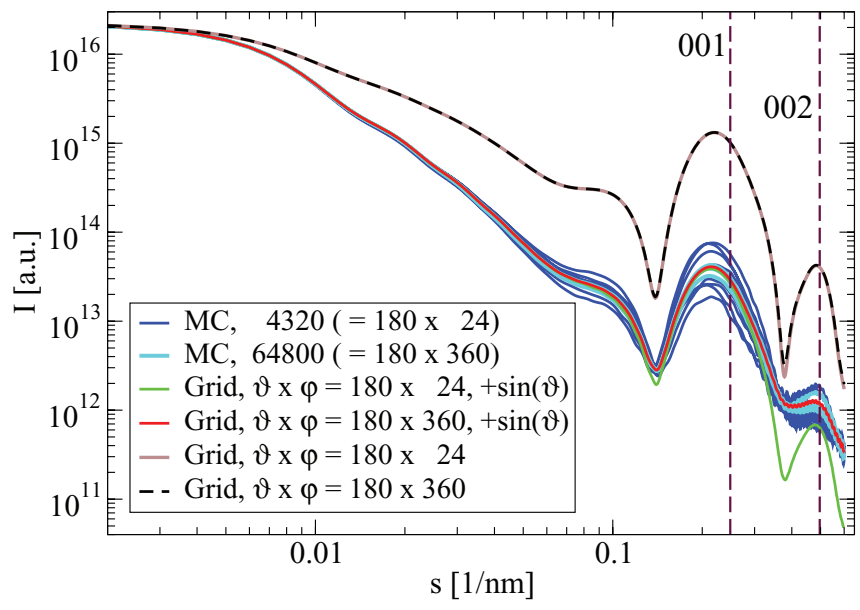

FIG. 11. (Color online) Simulated SAXS powder patterns for 100 tripalmitin platelets with statistically varying diameters and thicknesses for different spherical codes and point densities (i.e., number of orientations). For all tested spherical codes the particles are exactly the same. Patterns obtained with the Monte Carlo (MC) method spreading the orientations randomly on a sphere are taken as reference. Only if the $\sin \vartheta$ weighting factor is included with the grid algorithm is a reasonable agreement with the MC curves obtained.

algorithms to counterbalance the higher point density at the poles (crowding) that emerges when using the grid algorithm. However, if the grid algorithm is applied with the $\sin \vartheta$ weighting factor that prevents the crowding around the poles (green and red curve) a good agreement with the Monte Carlo algorithm is obtained. Hence, in the new program XNDIFF the weights $w_{v}=\sin \vartheta_{v}$ are now implemented and used by default when using the grid algorithm. By this, Eq. (B18) can be rewritten for the grid algorithm as

$$
\Upsilon_{j}(s)=\sum_{n=1}^{M}\left(\frac{\sum_{v=1}^{N_{\vartheta}}\left[\sin \vartheta_{v} \sum_{u=1}^{N_{\varphi}} \xi_{j}\left(\mathbf{Q}_{u v}\right)\right]}{\sum_{v=1}^{N_{\vartheta}} \sin \vartheta_{v}}\right),
$$

with the normalization $\sum_{v=1}^{N_{\vartheta}} \sin \vartheta \simeq N_{\vartheta}\langle\sin \vartheta\rangle_{\vartheta}$.

From Fig. 11 it can be also observed that the convergence of the grid algorithm is up to the range of tripalmitin's $001 \mathrm{Bragg}$ reflection nearly independent of the number of points along the azimuthal direction, $\varphi$ (see green vs red curve), it actually turns out that in this $s$ range only a sufficiently fine resolution for the polar polar angle $\vartheta$ is important. For simulations including also higher scattering angles (about $s>0.3 \mathrm{~nm}^{-1}$ ) a fine sampling of the azimuthal direction $\varphi$ is necessary.

By contrast, as compared with the grid algorithm the Monte Carlo algorithm exhibits over a wide part of the $s$ range a significantly slower convergence for the same total number of points on the orientation sphere (blue curves with 4320 points vs cyan curves with 64800 points). By this, also for the range $0.03<s<0.3 \mathrm{~nm}^{-1}$ of the order of $10^{5}$ orientations are necessary to obtain convergent results. Thus, a practical application of the Monte Carlo algorithms for the powder average seems rather inappropriate. It should be noted that other efficient spherical codes like REPULSION [72] or SPIRAL [73] were not implemented. However, as a result of other tests [74] it seems unrealistic to gain any 
significant improvements in performance as compared with the implemented grid algorithm.

To conclude, for the tripalmitin suspensions studied here the grid algorithm with the $\sin \vartheta$ weighting factor provides correct results and a superior performance as compared to the Monte Carlo algorithms. For the present study the simulations are run with the grid algorithm using point densities of $N_{\vartheta} \times$ $N_{\varphi}=180 \times 360$ corresponding to a grid on the upper $\mathbb{S}^{2}$ with $\vartheta \in\left[0.25^{\circ}, 89.75^{\circ}\right]$ in steps of $0.5^{\circ}$ and $\varphi \in\left[0.5^{\circ}, 359.5^{\circ}\right]$ in steps of $1^{\circ}$.

If one would restrict the simulations to a maximum $s$ of $0.3 \mathrm{~nm}^{-1}$, they could be run much faster by using lower point densities in the $\varphi$ direction (as it was usually done in the previous study [12]) without any loss in correctness.

\section{$e$. Lorentz and polarization corrections}

Powder samples consist of many crystallites with random orientation. However, only a fraction of the crystals has the proper orientation and will contribute to the diffracted Bragg intensity at the scattering angle $2 \theta$. One can show that this fraction is proportional to $\cos \theta$. Since for smallangle scattering $2 \theta$ is below $8^{\circ}$ this Lorentz factor can be neglected [75]. Furthermore, for typical SAXS measurements the polarization factor is close to 1 .

\section{Computational aspects}

The simulations were run on the high-performance computing cluster Woodcrest at the Regionales Rechenzentrum Erlangen (RRZE). The Woodcrest cluster provides about 200 nodes each equipped with two Xeon 5160 Woodcrest dual core chips (four cores per node) running at $3.0 \mathrm{GHz}, 8$ gigabytes of RAM shared memory and 4 megabytes shared L2 Cache per dual core. To exploit the full computing power, the computationally intensive code sections of XNDIFF, e.g., the ensemble and powder averages, are parallelized with the OpenMP API that supports shared memory multiprocessing programming for the $\mathrm{C}++$ code.

All simulations are carried out with a default volume fraction of the crystals of $\varphi_{\text {cry }}=0.01$; the real concentration of the measured suspension can be later adjusted by a simple scaling as indicated in Eq. (D4).

Especially simulations of particle stacks for concentrated tripalmitin suspensions [38] are computationally expensive because on the order of 100 stacks are necessary until the scattering patterns converge. A simulation run for an ensemble of 100 stacks of 5 particles (with platelet thicknesses up to 10 unit cells) for one parameter set $\left(d_{\text {isl }}, d_{\text {osl }}, R\right.$, and $\left.\sigma_{R}\right)$ using a $180 \times 360$ grid $(\theta \times \varphi)$ for the powder average takes about 12 $\mathrm{h}$ on one node. It should be noted that simultaneously also the scattering pattern for 100 stacks of 4,3 , and 2 particles each, as well as $5 \times 100=500$ individual particles with thicknesses of 1-10 unit cells are obtained. Parameter scans for 120 parameter sets for the shell thicknesses $d_{\text {isl }}$ and $d_{\text {osl }}$ (each thickness in steps of $2 \AA$ in the range $4 \leqslant d_{\text {isl }}+d_{\text {osl }} \leqslant 32 \AA$ ) with fixed parameters $R$ and $\sigma_{R}$ can be done typically within a day on the Woodcrest cluster.

For dilute suspensions, the computational efforts can be significantly reduced since in the considered $Q$ range (corresponding to sizes between about 1 and $100 \mathrm{~nm}$ ) the overall particle diameters are not that important. The scattering patterns of individual particles converge, therefore, already for small ensembles with less than ten particles. Consequently, the total computing time for one fixed parameter set drops down to less than half an hour on one node. When considering only individual platelets, similar parameter scans for $d_{\text {isl }}$ and $d_{\text {osl }}$ could be done on modern desktop machines with multicore CPUs within a few days.

\section{Fitting routine}

The routine that facilitates the simultaneous fitting of multiple datasets was written in MATHEMATICA. The most important fit parameters are the electron densities and neutron SLDs for the stabilizer layer, as well as the distribution of the linear combination coefficients $c_{i}$, representing the volume fractions of the $i$ th ensemble.

For both, SAXS and SANS, a constant background is added as a fit parameter.

The routine makes it possible to fix or to optimize parameters, specify ranges as well as constraints between parameters which was used in the models for the stabilizer layers in Sec. III C and Appendices F and G. A constraint that was always applied is given by the completeness relation in Eq. (D7) for the volume fractions $c_{i}$. However, for simultaneous fits and in particular with additional constraints it is necessary to relax this constraint, such that the sum over all $c_{i}$ may vary within bounds of $20 \%$ around 1 . Furthermore, a scaling factor $\chi$ for the simulated SAXS patterns was permitted within bounds of $25 \%$ around 1, accounting for possible errors in the absolute intensity scaling for the SAXS curves (e.g., due to errors in determining the precise thickness of the capillary) relative to the SANS data.

Using MATHEMATICA's built-in global optimization function NMinimize, different global optimization algorithms, namely Nelder-Mead (downhill simplex method), differential evolution, and simulated annealing, but also local optimizers (Levenberg-Marquardt, Conjugate gradient), were tested. The choice of the optimizer has only little influence on the fit results. For all further discussed fits the global simulated annealing algorithm was chosen, obviating the need to specify initial parameters.

For fits with many experimental datasets and/or modelbased fits for the stabilizer layer including many constraints (cf. Sec. III C), the computation time can increase dramatically. Parallelization of the code facilitates these fits even for about 100 simulated datasets as they are obtained, e.g., from a parameter scan for the thicknesses of the stabilizer layer within a few hours on a modern multicore CPU.

The objective function $T$ that has to be minimized is formulated as a least squares problem on a logarithmic scale, given by

$$
\begin{aligned}
T(\mathbf{y}, \mathbf{x}) & =\sum_{v}\left\|\ln \left[v\left(\frac{d \Sigma_{\text {mod }}}{d \Omega}\right)\right]-\ln \left[v\left(\frac{d \Sigma_{\exp }}{d \Omega}\right)\right]\right\|_{2}^{2} \\
& =\sum_{v}\left\|\ln \left[\frac{{ }^{v}\left(d \Sigma_{\text {mod }} / d \Omega\right)\left(s ; \mathbf{y},{ }^{v} \mathbf{x}\right)}{{ }^{v}\left(d \Sigma_{\exp } / d \Omega\right)(s)}\right]\right\|_{2}^{2},
\end{aligned}
$$

where $v$ runs over all available experimental datasets [e.g., two for a simultaneous fit of $\operatorname{SAXS}(v=1)$ and $\operatorname{SANS}(v=2)$ 
data] and the 2-norm applies to the vector of the intensity differences in the fitted $s$ range $\left(0.02<s<0.45 \mathrm{~nm}^{-1}\right.$ in this study). $\mathbf{y}$ denotes the fit parameters that are simultaneously fitted to all datasets (usually the linear combination coefficients $c_{i}$ if the same sample is measured; but may also include, for example, the contrasts for the shell, if multiple SANS patterns are fitted for samples sharing the same kind of phospholipid). The parameters in ${ }^{v} \mathbf{x}$ belong either solely to the $v$ th dataset or to a subset of all datasets. Furthermore, the parameters within and in between the $\mathbf{y}$ and ${ }^{v} \mathbf{x}$ might be coupled by constraints. The modeled scattering cross sections ${ }^{v}\left(d \Sigma_{\text {mod }} / d \Omega\right)$ are taken from Eq. (B16); the ${ }^{v}\left(d \Sigma_{\exp } / d \Omega\right)$ denote the experimental datasets.

\section{Synopsis}

This section is intended to give a short overview of the previously described simulation and fitting procedure.

For the $i$ th simulated ensemble and the $v$ th experimental dataset, the ten scattering cross section functions ${ }^{\nu} \Upsilon_{j}^{i}(s)$ $(j=1, \ldots, 10)$ can be obtained by the ensemble and powder average in Eq. (B18) from the functions ${ }_{n}^{v} \xi_{j}^{i}(\mathbf{Q})$ defined in Eqs. (C1) to (C4) for particle stacks and Eq. (C5) for individual particles in Appendix C. Normalization with the simulated sample volume in Eq. (B14) yields the ten macroscopic scattering cross section functions ${ }^{v} Y_{j}^{i}(s)(j=1, \ldots, 10)$ :

$$
{ }_{n}^{v} \xi_{j}^{i}(\mathbf{Q}) \stackrel{\langle\cdot\rangle_{\Omega_{\mathbf{Q}}},\left\langle\cdot{ }_{M^{i}}\right.}{\underset{(\mathrm{B} 18)}{\longrightarrow}}{ }^{v} \Upsilon_{j}^{i}(s) \underset{(\mathrm{B} 14)}{\stackrel{V^{i}, \varphi_{\mathrm{cry}}}{\longrightarrow}}{ }^{v} Y_{j}^{i}(s) .
$$

For each dataset $v$ and ensemble $i$ the computed ten colons of ${ }^{v} Y_{j}^{i}(s)$ are then stored in an ASCII file.

In the subsequent fitting procedure at first for each dataset $v$ and ensemble $i$ the ten colons for the ${ }^{v} Y_{j}^{i}(s)$ are loaded from the file and optionally convolved with an appropriate resolution function to regard instrumental smearing effects as discussed in more detail for SANS in Appendix E. The macroscopic scattering cross sections ${ }^{\nu}(d \Sigma / d \Omega)^{i}(s)$ are calculated in Eq. (B13) by summation over all ${ }^{v} Y_{j}^{i}$ with the contrast coefficients ${ }^{v} \Gamma_{j}$ for the dispersion medium and stabilizer layer defined in Eqs. (B12) and (B9).

In the next step for each dataset $v$ the linear combination of all ${ }^{\nu}(d \Sigma / d \Omega)^{i}(s)$ with the coefficients $c_{i}$ is calculated in Eq. (B16) to obtain the modeled macroscopic scattering cross section ${ }^{\nu}(d \Sigma / d \Omega)(s)$. Finally, the objective function value $T$ that has to be minimized is computed by summing over the least squares of all datasets:

$$
{ }^{v} Y_{j}^{i}(s) \underset{(\mathrm{B} 13)}{\stackrel{\sum_{j}{ }^{v} \Gamma_{j}}{\longrightarrow}} v\left(\frac{d \Sigma}{d \Omega}\right)^{i}(s) \underset{(\mathrm{B} 16)}{\stackrel{\sum_{i} c_{i}}{\longrightarrow}} v\left(\frac{d \Sigma}{d \Omega}\right)(s) \underset{(\mathrm{B} 22)}{\stackrel{\sum_{v}}{\longrightarrow}} T .
$$

The fit procedure yields the electron densities and/or neutron SLD given in Eqs. (B3) via the contrasts $\Delta \rho_{j}$ in Eqs. (B9) from the $\Gamma_{j}$ in Eq. (B12) and, furthermore, the thickness distribution $\left(c_{i}\right)$ for the platelets. Optimization of geometrical parameters like the shell thicknesses $d_{\text {isl }}$ and $d_{\text {osl }}$, the particle diameter distribution $\left[\mu_{D}, \sigma_{D}\right.$ in Eq. (I1)] or the distribution for the interparticle distances in stacks $\left(R, \sigma_{R}\right)$ still require time-consuming parameter scans. However, because of the code improvements and the use of HPC clusters such parameter scans are now feasible within reasonable time.

\section{APPENDIX C: LIST OF THE FUNCTIONS $\boldsymbol{\xi}_{j}$}

The functions ${ }_{n} \xi_{j}$ used in Eq. (B18) can be directly derived from Eq. (B10) in a straightforward calculation assuming real valued scattering contrasts for the stabilizer layer and dispersion medium (far away from any resonance related absorptions). For particle stacks typically present in concentrated $(>4 \%)$ triglyceride suspensions [7] the expressions in Eqs. (C1) to (C4) have to be used, for individual particles Eqs. $(\mathrm{C} 1)$ to $(\mathrm{C} 4)$ reduce to Eq. $(\mathrm{C} 5)$. The scattering contributions from the same kind of shell of the particles in a stack [Eq. (C1)] and the interferences between different shells [in the same particle or in another particles in the stack, Eq. (C2)] can be written as

$$
\begin{gathered}
{ }_{n} \xi_{j}(\mathbf{Q})=\sum_{k=1}{ }_{n}^{M}\left[\left|{ }_{n} P_{k, j}\right|^{2}+2 \sum_{l=1}^{k-1} \operatorname{Re}\left\{{ }_{n} P_{k, j} \overline{{ }_{n} P_{l, j}} e^{\left.i \mathbf{Q} \cdot{ }_{n} \mathbf{R}_{k}-{ }_{n} \mathbf{R}_{l}\right)}\right\}\right], \quad j=1,2,3, \\
{ }_{n} \xi_{j}(\mathbf{Q})=2 \cdot \sum_{k=1}^{n M}\left[\operatorname{Re}\left\{{ }_{n} P_{k, j_{1}} \overline{{ }_{n} P_{k, j_{2}}}\right\}+\sum_{l=1}^{k-1} \operatorname{Re}\left\{\left({ }_{n} P_{k, j_{1}} \overline{{ }_{n} P_{l, j_{2}}}+{ }_{n} P_{k, j_{2}} \overline{{ }_{n} P_{l, j_{1}}}\right) e^{i \mathbf{Q} \cdot\left({ }_{n} \mathbf{R}_{k}-{ }_{n} \mathbf{R}_{l}\right)}\right\}\right], \quad j=4,5,6,
\end{gathered}
$$

where $\left(j_{1}, j_{2}\right)$ equals $(3,2)$ for $j=4,(3,1)$ for $j=5$, and $(2,1)$ for $j=6$. The interference terms between all shells and all crystal cores are given by

$$
{ }_{n} \xi_{j}(\mathbf{Q})=-2 \cdot \sum_{k=1}^{{ }_{n} M}\left[\operatorname{Re}\left\{{ }_{n} F_{k} G_{k} \overline{{ }_{n} P_{k, j}}\right\}+\sum_{l=1}^{k-1} \operatorname{Re}\left\{\left({ }_{n} F_{k}{ }_{n} G_{k} \overline{{ }_{n} P_{l, j}}+\overline{{ }_{n} F_{l n} G_{l n}} P_{k, j}\right) e^{i \mathbf{Q} \cdot\left({ }_{n} \mathbf{R}_{k}-{ }_{n} \mathbf{R}_{l}\right)}\right\}\right], \quad j=7,8,9
$$

Finally, the scattering contributions from the crystal cores in the stacks and the interferences between the crystal cores in the stacks can be written as

$$
{ }_{n} \xi_{10}(\mathbf{Q})=\sum_{k=1}^{{ }_{n} M}\left[\left|{ }_{n} F_{k}\right|^{2}\left|{ }_{n} G_{k}\right|^{2}+2 \sum_{l=1}^{k-1} \operatorname{Re}\left\{{ }_{n} F_{k n} G_{k} \overline{{ }_{n} F_{l n} G_{l}} e^{i \mathbf{Q} \cdot\left({ }_{n} \mathbf{R}_{k}-{ }_{n} \mathbf{R}_{l}\right)}\right\}\right]
$$


Since the current code implementation allows only particle assemblies with platelets lying parallel to each other, the structure amplitudes $F$ in the expressions for the ${ }_{n} \xi_{j}$ become actually independent of the indices $l$ and $k$.

For suspensions with only ensembles of individual particles $\left({ }_{n} M=1, \forall n\right)$ the expressions for the ${ }_{n} \xi_{j}$ reduce to

$$
{ }_{n} \xi_{j}(\mathbf{Q})= \begin{cases}\left|{ }_{n} P_{1, j}\right|^{2}, & j=1,2,3, \\ 2 \cdot \operatorname{Re}\left\{{ }_{n} P_{1, j_{1}} \overline{{ }_{n} P_{1, j_{2}}}\right\}, & j=4,5,6, \\ -2 \cdot \operatorname{Re}\left\{{ }_{n} F_{1}{ }_{n} G_{1} \overline{P_{1, j}}\right\}, & j=7,8,9, \\ \left|{ }_{n} F_{1}\right|^{2}\left|{ }_{n} G_{1}\right|^{2}, & j=10,\end{cases}
$$

with the same values for $\left(j_{1}, j_{2}\right)$ as mentioned before.

\section{APPENDIX D: INTERPRETATION OF THE LINEAR COMBINATION COEFFICIENTS $c_{i}$}

This section discusses the physical meaning of the linear coefficients $c_{i}$ in Eq. (B16). The following considerations will be carried out at first for suspensions of solely individual particles, i.e., without any particle assemblies $\left({ }_{n} M^{i}=1, \forall i, n\right)$. As mentioned at the end of the section, the considerations are also valid for particle assemblies. The dilute tripalmitin suspensions considered in this study are mixtures of platelets with different thicknesses, expressed by the number $i$ of unit cells of tripalmitin. An example of the situation is sketched in Fig. 12 for a dilute dispersion (D) of individual nanocrystals with thicknesses of up to 3 unit cells. For such a mixture the

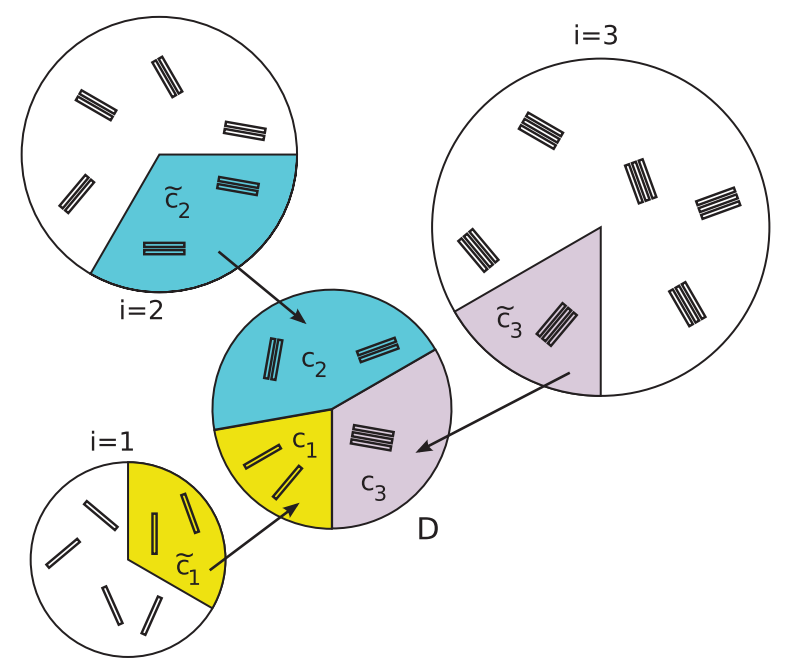

FIG. 12. (Color online) Sketch for a dilute dispersion (D) with concentration $\varphi_{\text {cry }}$ comprising of individual platelets with thicknesses of 1,2 , and 3 unit cells. The other three spheres represent the simulated ensembles $i$ of platelets with thicknesses of $i$ unit cells. As in a typical simulation all ensembles possess the same concentration $\varphi_{\text {cry }}$ and the same number of members $M^{i}$. The sphere volumes represent the associated dispersion volumes corresponding to $\varphi_{\text {cry }}$. For the sake of simplicity the lateral platelet diameters are assumed in the drawing to be the same for all platelets; in a real suspension and in the simulation runs the diameters are subject to a statistical distribution. For this simplified exemplary suspension the volume fractions of the three ensembles are $c_{1}=2 / 9, c_{2}=4 / 9$, and $c_{3}=1 / 3$, respectively. For the simulated ensembles this corresponds to volume fractions of $\tilde{c}_{1}=1 / 3, \tilde{c}_{2}=1 / 3$, and $\tilde{c}_{3}=1 / 6$, respectively. overall macroscopic scattering cross section $(d \Sigma / d \Omega)$ can be written as the weighted sum of the scattering cross sections $(d \sigma / d \Omega)^{i}$ of all ensembles normalized by the total volume of the dispersion. The dispersion volume $V_{D}$ contains all fractions of the ensembles, i.e., $\tilde{c}_{i} \cdot\left(V^{i} / \varphi_{\text {cry }}\right)$ for ensemble $i$ :

$$
\left(\frac{d \Sigma}{d \Omega}\right)=\frac{1}{V_{D}} \sum_{i} \tilde{c}_{i} \cdot\left(\frac{d \sigma}{d \Omega}\right)^{i}=\frac{\sum_{i} \tilde{c}_{i} \cdot(d \sigma / d \Omega)^{i}}{\sum_{i} \tilde{c}_{i}\left(V^{i} / \varphi_{\text {cry }}\right)} .
$$

The scattering cross sections for the crystals in each ensemble $i$ are given by the sum over the scattering cross sections of all $M^{i}$ crystals and $V^{i}$ is the sum of all crystal volumes in the ensemble $i$ :

$$
\left(\frac{d \sigma}{d \Omega}\right)^{i}(s)=\sum_{n=1}^{M^{i}} n\left(\frac{d \sigma}{d \Omega}\right)^{i}(s), \quad V^{i}=\sum_{n=1}^{M^{i}} V^{i} .
$$

The $\tilde{c}_{i}\left(0 \leqslant \tilde{c}_{i}<\infty\right)$ can be considered as the volume fraction taken from the $i$ th ensemble for the dispersion $D$. For large enough ensembles $\left(M^{i} \rightarrow \infty\right)$ and a symmetrical distribution function for the platelet diameters, the $\tilde{c}_{i}$ can be regarded as the number fraction of particles taken from the ensemble $i$ and, thus, $\tilde{c}_{i} / V_{D}$ as the number density of particles from ensemble $i$ in the dispersion.

The scattering cross sections $(d \sigma / d \Omega)^{i}$ of the $i$ th ensemble are not computed in the simulations as they are required in the linear combination in Eq. (D1) but the macroscopic ones $(d \Sigma / d \Omega)^{i}$ are. Therefore, a mixture of ensembles in a dispersion corresponds to a linear combination similar to the one in Eq. (B16),

$$
\left(\frac{d \Sigma}{d \Omega}\right)(s)=\sum_{i} c_{i}\left(\frac{d \Sigma}{d \Omega}\right)^{i}(s) .
$$

Bearing in mind that

$$
\left(\frac{d \Sigma}{d \Omega}\right)^{i}(s)=\frac{\varphi_{\text {cry }}}{V^{i}}\left(\frac{d \sigma}{d \Omega}\right)^{i}(s)
$$

and expanding Eq. (D1) as

$$
\left(\frac{d \Sigma}{d \Omega}\right)(s)=\frac{\sum_{i}\left\{\tilde{c}_{i}\left(V^{i} / \varphi_{\text {cry }}\right)\left[\frac{(d \sigma / d \Omega)^{i}(s)}{\left(V^{i} / \varphi_{\text {cry }}\right)}\right]\right\}}{\sum_{i}\left\{\tilde{c}_{i}\left(V^{i} / \varphi_{\text {cry }}\right)\right\}},
$$

the $c_{i}$ can be identified by comparing Eqs. (D3) and (D5) as

$$
c_{i}=\frac{\tilde{c}_{i}\left(V^{i} / \varphi_{\text {cry }}\right)}{\sum_{j} \tilde{c}_{j}\left(V^{j} / \varphi_{\text {cry }}\right)}=\frac{\tilde{c}_{i} V^{i}}{\sum_{j} \tilde{c}_{j} V^{j}} .
$$

Therefore, the linear coefficients $c_{i}$ have the meaning of volume fractions with $0 \leqslant c_{i} \leqslant 1$. Each $c_{i}$ represents the volume fraction of dispersion of ensemble $i$ in the total dispersion $\mathrm{D}$ (or equivalently the volume fraction of crystals from ensemble $i$ with respect to the total crystal volume of all ensembles in D). Furthermore, Eq. (D6) imposes a completeness relation for the coefficients $c_{i}$,

$$
\sum_{i} c_{i}=1
$$

which should be regarded as a constraint in the fitting procedure.

For concentrated tripalmitin suspensions, e.g., particle stacks also can be present. Nevertheless, similar considerations 
lead to the same interpretation, namely that the $c_{i}$ correspond to the volume fractions of the ensemble $i$, representing typically stacks with a specific number of platelets in the suspensions. The expression for the volume $V^{i}$ of ensemble $i$ in Eq. (D2) has then to be replaced by the double sum $V^{i}=\sum_{n=1}^{M^{i}} \sum_{k=1}^{{ }_{n} M^{i}} V_{k}^{i}$ over all stacks in the ensemble $i$ and all particles $k$ in the $n$th stack.

\section{APPENDIX E: SMEARING OF SANS DATA}

For SANS experiments (typically done in a pinhole geometry) three contributions for smearing can be observed [76,77]. As for (pinhole) SAXS, the divergence of the beam may lead to considerable smearing at very small scattering angles. In contrast to SAXS, for SANS also at high $Q$ smearing has to be taken into account due to the wavelength spread of the incoming neutron beam. Furthermore, the finite resolution of the detector gives rise to smearing effects.

Because of the linear character of the operations in Eqs. (B16) and (B13) and because of convolution integrals, the smearing can be taken into account for each ensemble $i$ as a convolution of the ten functions $Y_{j}^{i}$ from the simulations with an appropriate kernel $K$

$$
Y_{j}^{i}(s)=S * Y_{j}^{i}(s) \approx \int_{s-\Delta s}^{s+\Delta s} K\left(s, s^{\prime}\right) \cdot Y_{j}^{i}\left(s^{\prime}\right) d s^{\prime} .
$$

Here, $\Delta s$ was chosen as the $3 \sigma$ interval $3 \sigma_{s}$, with $\sigma_{s}$ being given by for the first two contributions as [77]

$$
\begin{aligned}
\sigma_{s}^{2}(s) & =\left[\frac{s}{2 \sqrt{2 \ln 2}} \cdot\left(\frac{\Delta \lambda}{\lambda}\right)\right]^{2}+\left[\left(\frac{2}{\lambda}\right)^{2}-s^{2}\right] \Delta \theta^{2} \\
& \approx\left[\frac{s}{2 \sqrt{2 \ln 2}} \cdot\left(\frac{\Delta \lambda}{\lambda}\right)\right]^{2} .
\end{aligned}
$$

The latter approximation neglects the effects of the beam divergence and is a good approximation for the medium and high $Q$ range $\left(s>0.03 \mathrm{~nm}^{-1}\right)$. The smearing of the simulated SANS curves improves the fit results around the $001 \mathrm{Bragg}$ reflection due to an additional broadening effect.

The convolution kernel in Eq. (E1) for the smearing induced by the wavelength spread is assumed to be a Gaussian function,

$$
K\left(s, s^{\prime}\right)=\frac{1}{\sqrt{2 \pi \sigma_{s}^{2}(s)}} \exp \left[-\frac{\left(s-s^{\prime}\right)^{2}}{2 \sigma_{s}^{2}(s)}\right] .
$$

For the exposures considered in this study, a wavelength spread of $20 \%$ was used. Therefore, all simulated SANS patterns were smeared in the high $Q$ range before the fitting procedure with the help of Eqs. (E1) and (E3) using a $\Delta \lambda / \lambda=0.2$.

\section{APPENDIX F: FIT CONSTRAINTS FOR MODEL B}

In this section the fit constraints for the scattering contrasts of the molecular model B discussed in Sec. III C are derived.

Assuming that all head groups are situated completely in the outer shell and all chains in the inner shell, the electron densities for the inner and outer shells can be written as

$$
\begin{gathered}
\rho_{\text {isl }}^{X}=\varphi_{c h} \rho_{c h}^{X}+\left(1-\varphi_{c h}\right) \rho_{d m}^{X}, \\
\rho_{\text {osl }}^{X}=\varphi_{h} \rho_{h}^{X}+\left(1-\varphi_{h}\right) \rho_{d m}^{X},
\end{gathered}
$$

where $\varphi_{c h}$ and $\varphi_{h}$ denote the volume fractions and $\rho_{c h}^{X}$ and $\rho_{h}^{X}$ denote the electron densities of the acyl chains in the inner and head groups in the outer shell, respectively. Similar expressions can be obtained for the neutron SLD's $\rho_{\text {isl }}^{n}$ and $\rho_{\text {osl }}^{n}$ by using the same volume fractions $\varphi_{c h}$ and $\varphi_{h}$ but replacing the electron densities in Eqs. (F1) and (F2) with their neutron SLD counterparts,

$$
\begin{gathered}
\rho_{\text {isl }}^{n}=\varphi_{c h} \rho_{c h}^{n}+\left(1-\varphi_{c h}\right) \rho_{d m}^{n}, \\
\rho_{\text {osl }}^{n}=\varphi_{h} \rho_{h}^{n}+\left(1-\varphi_{h}\right) \rho_{d m}^{n} .
\end{gathered}
$$

The values for $\rho_{c h}^{X}, \rho_{h}^{X}$ and $\rho_{c h}^{n}, \rho_{h}^{n}$ for a DOPC molecule can be found in Table II.

The third constraint respects the molecular volumes of the phospholipid head groups and acyl chains and relates the outer shell with the inner shell at their shared interface $S$. The following condition must be fulfilled at the interface:

$$
S=\frac{V_{\text {isl }}}{d_{\text {isl }}}=\frac{V_{\text {osl }}}{d_{\text {osl }}} \Rightarrow \frac{V_{\text {isl }}}{V_{\text {osl }}}=\frac{d_{\text {isl }}}{d_{\text {osl }}} .
$$

If $N$ phospholipid molecules reside in the stabilizer layer, the volume of the inner shell $V_{\text {isl }}$ can be expressed as $\varphi_{c h} V_{\text {isl }}=$ $N \cdot V_{c h}$ and similarly for the outer shell, $\varphi_{h} V_{\text {osl }}=N \cdot V_{h}$. The volumes of the acyl chains $V_{c h}$ and the head group $V_{h}$ for one phospholipid molecule are tabulated for DOPC in Table II. Thus, the latter condition can be reformulated as

$$
\frac{V_{c h}}{V_{h}} \cdot \frac{\varphi_{h}}{\varphi_{c h}}=\frac{d_{\text {isl }}}{d_{\text {osl }}} .
$$

For a consistent model all three constraints should be fulfilled simultaneously. The volume fraction $\varphi_{c h}$ in Eqs. (F1) and (F3) and similarly $\varphi_{h}$ in Eqs. (F2) and (F4) for the x-ray and neutron SLDs must be the same, respectively. Furthermore, Eq. (F6) establishes a connection between $\varphi_{h}$ and $\varphi_{c h}$. With these three constraints it is possible to reduce the number of fitted contrasts from five for the unconstrained problem (model A) to only two. Choosing $\rho_{d m}^{X}$ and $\rho_{\text {isl }}^{X}$ as independent fit parameters, the linear equation system of Eqs. (F1) to (F6) can be solved for the remaining three parameters $\rho_{\mathrm{isl}}^{n}, \rho_{\mathrm{osl}}^{n}$, and $\rho_{\mathrm{osl}}^{X}$,

$$
\begin{aligned}
& \rho_{\mathrm{isl}}^{n}=\frac{\rho_{d m}^{X} \rho_{c h}^{n}-\rho_{c h}^{X} \rho_{d m}^{n}+\rho_{\mathrm{isl}}^{X} \cdot \delta \rho_{d m, c h}^{n}}{\delta \rho_{d m, c h}^{X}}, \\
& \rho_{\mathrm{osl}}^{n}=\rho_{d m}^{n}-\frac{d_{\mathrm{isl}}}{d_{\mathrm{osl}}} \cdot \frac{V_{h}}{V_{c h}} \frac{\delta \rho_{d m, i s l}^{X}}{\delta \rho_{d m, c h}^{X}} \cdot \delta \rho_{d m, h}^{n}, \\
& \rho_{\mathrm{osl}}^{X}=\rho_{d m}^{X}-\frac{d_{\mathrm{isl}}}{d_{\mathrm{osl}}} \cdot \frac{V_{h}}{V_{c h}} \frac{\delta \rho_{d m, i s l}^{X}}{\delta \rho_{d m, c h}^{X}} \cdot \delta \rho_{d m, h}^{X},
\end{aligned}
$$

where $\delta \rho_{i, j}$ denotes the contrasts $\delta \rho_{i, j}=\rho_{i}-\rho_{j}$.

In order to obtain volume fractions between 0 and 1 , the $\rho_{\text {isl }}^{X / n}$ and $\rho_{\text {osl }}^{X / n}$ have to be restricted. $\rho_{d m}^{X}$ and $\rho_{\text {isl }}^{X}$ were allowed to vary within the bounds $\left[\rho_{d m, \ominus}^{X}, \rho_{d m, \oplus}^{X}\right]$ and $\left[\rho_{c h}^{X}, \rho_{d m, \oplus}^{X}\right]$, respectively, with $\rho_{d m, \ominus}^{X}=333 \mathrm{~nm}^{-3}$ and $\rho_{d m, \oplus}^{X}=345 \mathrm{~nm}^{-3}$. $\rho_{\text {isl }}^{n}, \rho_{\text {osl }}^{n}$, and $\rho_{\text {osl }}^{X}$ were restricted to the intervals $\left[\rho_{c h}^{n}, \rho_{d m}^{n}\right]$, $\left[\rho_{h}^{n}, \rho_{d m}^{n}\right]$, and $\left[\rho_{d m, \ominus}^{X}, \rho_{h}^{X}\right]$, respectively. The electron densities and neutron SLDs of the molecules or molecule fragments can be found Table II.

The volume fraction of the head groups in the outer layer $\varphi_{h}$ and the volume fraction of the chains in the inner layer $\varphi_{c h}$ 
can be calculated by

$$
\varphi_{h}=\frac{\rho_{d m}^{X}-\rho_{\mathrm{ol}}^{X}}{\rho_{d m}^{X}-\rho_{h}^{X}}, \quad \varphi_{c h}=\frac{\rho_{d m}^{X}-\rho_{\mathrm{isl}}^{X}}{\rho_{d m}^{X}-\rho_{c h}^{X}} .
$$

\section{APPENDIX G: FIT CONSTRAINTS FOR MODEL D}

Similarly as discussed for model B in Appendix F, three constraints can be formulated for model D. While Eqs. (F2) and (F4) for the outer shell stay the same as for model B, for the inner shell, which cosists now of acyl chains and full DOPC molecules, $\rho_{d m}^{X}$ and $\rho_{d m}^{n}$ have to be replaced in Eqs. (F1) and (F3) with $\rho_{\text {DOPC }}^{X}$ and $\rho_{\text {DOPC }}^{n}$, respectively.

With the volume of $N_{1}$ DOPC molecules that are completely in the inner shell $\left(1-\varphi_{c h}\right) V_{\text {isl }}=N_{1} V_{\text {DOPC }}$, the chain volume of $N_{2}$ DOPC molecules that are spread over both shells $\varphi_{c h} V_{\text {isl }}=N_{2} V_{c h}$, and correspondingly the head group volume of those $N_{2}$ DOPC molecules in the outer shell $\varphi_{h} V_{\text {osl }}=N_{2} V_{h}$ and the volume of water in the outer shell $\left(1-\varphi_{h}\right) V_{\text {osl }}=V_{d m}$ one can write the third constraint from Eq. (F5) as

$$
\frac{d_{\text {isl }}}{d_{\text {osl }}}=\frac{V_{\text {isl }}}{V_{\text {osl }}}=\frac{N_{1} V_{\mathrm{DOPC}}+N_{2} V_{c h}}{N_{2} V_{h}+V_{d m}}=\frac{V_{c h}}{V_{h}} \cdot \frac{\varphi_{h}}{\varphi_{c h}} .
$$

As for model B, $\rho_{\text {isl }}^{n}, \rho_{\text {osl }}^{n}$, and $\rho_{\text {osl }}^{X}$ can be expressed as functions of $\rho_{\text {isl }}^{X}$ and $\rho_{d m}^{X}$,

$$
\begin{gathered}
\rho_{\mathrm{isl}}^{n}=\frac{\rho_{\mathrm{DOPC}}^{X} \rho_{c h}^{n}-\rho_{c h}^{X} \rho_{\mathrm{DOPC}}^{n}+\rho_{\mathrm{isl}}^{X} \cdot \delta \rho_{\mathrm{DOPC}, \mathrm{ch}}^{n},}{\delta \rho_{\mathrm{DOPC}, \mathrm{ch}}^{X}}, \\
\rho_{\mathrm{osl}}^{n}=\rho_{d m}^{n}-\frac{d_{\mathrm{isl}}}{d_{\mathrm{osl}}} \cdot \frac{V_{h}}{V_{c h}} \frac{\delta \rho_{\mathrm{DOPC}, \mathrm{isl}}^{X}}{\delta \rho_{\mathrm{DOPC}, \mathrm{ch}}^{X}} \cdot \delta \rho_{d m, h}^{n}, \\
\rho_{\mathrm{osl}}^{X}=\rho_{d m}^{X}-\frac{d_{\mathrm{isl}}}{d_{\mathrm{osl}}} \cdot \frac{V_{h}}{V_{c h}} \frac{\delta \rho_{\mathrm{DOPC}, \mathrm{ssl}}^{X}}{\delta \rho_{\mathrm{DOPC}, \mathrm{ch}}^{X}} \cdot \delta \rho_{d m, h}^{X} .
\end{gathered}
$$

In the fitting procedure the parameter ranges were the same as for model B, except for the contrasts of the inner stabilizer layer where the upper limits are now governed by DOPC instead of the dispersion medium, i.e., $\rho_{\mathrm{isl}}^{X} \in\left[\rho_{c h}^{X}, \rho_{\mathrm{DOPC}}^{X}\right]$ and $\rho_{\text {isl }}^{n} \in\left[\rho_{c h}^{n}, \rho_{\text {DOPC }}^{n}\right]$. For the second fraction of phospholipids $\varphi_{h}$ and $\varphi_{c h}$ can be calculated with Eq. (F10) by replacing $\rho_{d m}^{X}$ with $\rho_{\text {DOPC }}^{X}$ in the equation for $\varphi_{c h}$. Using these volume fractions and Eq. (G1), the number ratio of phospholipids from groups No. 1 and No. 12 can be determined with

$$
\frac{N_{1}}{N_{2}}=\frac{V_{\mathrm{DOPC}}}{V_{c h}} \cdot \frac{\varphi_{c h}}{1-\varphi_{c h}}=\frac{V_{\mathrm{DOPC}}}{V_{h}} \cdot \frac{\varphi_{h}}{1-\varphi_{c h}} \cdot \frac{d_{\mathrm{isl}}}{d_{\mathrm{osl}}} .
$$

\section{APPENDIX H: VALIDITY TESTS OF THE SIMULATION METHOD}

Two simple tests were done to test the reliability of the simulation program.

The first test checks whether in the limit $Q \rightarrow 0$ the correct macroscopic scattering cross section value is obtained. Therefore, the powder average of just one tripalmitin platelet with given dimensions ${ }_{1} N_{1, \mu}$ (e.g., 100 in both, $\mathbf{a}_{1}$ and $\mathbf{a}_{2}$ direction and a thickness of 4 unit cells in $\mathbf{a}_{3}$ ([001] direction), stabilizer layer thicknesses, scattering contrasts $\Delta \rho_{j}$, and concentration $\varphi_{\text {cry }}=1$ is simulated. In this case the structure amplitude in Eq. (B2) reduces with Eq. (B4) to sums over all atomic numbers $Z_{l}$ or scattering lengths $b_{l}$ in the unit cell, ${ }_{1} F_{1}(0)=r_{e} \sum_{l} Z_{l}=r_{e} \cdot 904=2547.38 \mathrm{fm}$ for SAXS and ${ }_{1} F_{1}(0)=\sum_{l} b_{l}=14.68 \mathrm{fm}$ for SANS, respectively. The lattice amplitude in Eq. (B5) reduces to the product of all crystal dimensions, ${ }_{1} G_{1}(0)=\prod_{\mu 1} N_{1, \mu}$, and similarly the functions ${ }_{n} P_{k, j}$ in Eq. (B7) reduce to the volumes ${ }_{1} P_{1, j}(0)=$ ${ }_{1} V_{1, j}$. The crystal volume is given by ${ }_{1} V_{1,1}=V_{u c} \prod_{\mu}{ }_{1} N_{1, \mu}$ with $V_{u c}=\operatorname{det}\left(\mathbf{a}_{1}, \mathbf{a}_{2}, \mathbf{a}_{3}\right)=2.58 \mathrm{~nm}^{3}$. Finally the theoretical macroscopic scattering cross section in the limit $Q \rightarrow 0$ can be written as

$$
\left(\frac{d \Sigma}{d \Omega}\right)(0)=\frac{\varphi_{\text {cry }}}{{ }_{1} V_{1,1}}\left|{ }_{1} F_{1}(0){ }_{1} G_{1}(0)-\left(\sum_{j} \Delta \rho_{j 1} P_{1, j}(0)\right)\right|^{2},
$$

with the aforementioned expressions. Both, $\mathrm{x}$-ray and neutron simulations give very good agreement with the theoretical values that can be obtained with the last equation.

For the second test the integrated 001 Bragg reflection intensity was checked for the simulated $\mathrm{x}$-ray and neutron powder patterns of a macroscopic tripalmitin crystal (dimensions ${ }_{1} N_{1, \mu}$, volume $V=N \cdot V_{u c}$ with $\left.N=\prod_{\mu} N_{1, \mu}\right)$ in vacuum without a stabilizer layer (contrasts for the stabilizer layer are set to vacuum). Theoretically, the integrated $00 \mathrm{l}$ Bragg reflection intensity that XNDIFF should yield can be calculated as an integration over all orientations of $\mathbf{Q}$ in the upper half sphere and an integration over the modulus $Q$ that takes place in a small band with thickness $\Delta Q_{00 l}$ around $Q_{00 l}$,

$$
\left(\frac{d \Sigma}{d \Omega}\right)^{\mathrm{int}}\left(Q_{00 l}\right)=\frac{1}{V} \frac{\int_{2 \pi} \int_{\Delta Q_{00 l}}\left|F\left(\mathbf{Q}_{00 l}\right) G\left(\mathbf{Q}_{00 l}\right)\right|^{2} d^{3} \mathbf{Q}}{\int_{2 \pi} \int_{\Delta Q_{00 l}} d^{3} \mathbf{Q}} .
$$

For a large enough crystal providing sharp enough peaks at $\mathbf{Q}_{00 l}\left(\Delta Q_{00 l} \rightarrow 0\right)$, the denominator reduces virtually to a surface integral over the upper half sphere, $\int_{2 \pi} \int_{\Delta Q_{00 l}} d^{3} \mathbf{Q} \rightarrow$ $2 \pi \cdot Q_{00 l}^{2}$. As the lattice amplitude becomes a very sharp peak function for sufficiently large crystals, the structure amplitude $F\left(\mathbf{Q}_{00 l}\right)=F_{00 l}$ can be pulled out of the integral. Furthermore, with

$$
\int_{-\pi}^{\pi} \frac{\sin ^{2} \frac{1 N_{1, \mu} x_{\mu}}{2}}{\sin ^{2} \frac{x_{\mu}}{2}} d x_{\mu}=2 \pi \cdot{ }_{1} N_{1, \mu}, \quad x_{\mu}=\mathbf{Q}_{00 l} \cdot{ }_{1} \mathbf{a}_{1, \mu},
$$

the integral over the remaining lattice factor reduces to $N$. $(2 \pi)^{3} / V_{u c}$ and one obtains

$$
\left(\frac{d \Sigma}{d \Omega}\right)^{\text {int }}\left(Q_{00 l}\right)=\frac{N}{V} \frac{1}{2 \pi Q_{00 l}^{2}} \frac{(2 \pi)^{3}}{V_{u c}}\left|F_{00 l}\right|^{2}=\frac{\left|F_{00 l}\right|^{2}}{V_{u c}^{2} \cdot s_{00 l}^{2}} .
$$

This is also in accordance with the result derived in [75] for the integrated intensity of a powder ring of a Bragg reflection $h k l$ of a crystallite with $N$ unit cells,

$$
I_{\text {powder }}^{\text {int }}\left(Q_{h k l}\right)=\frac{N}{V} \frac{m_{h k l}}{4 \pi Q_{h k l}^{2}} \frac{(2 \pi)^{3}}{V_{u c}}\left|F_{h k l}\right|^{2},
$$

which reduces for $00 l$ reflections with multiplicities $m_{00 l}=2$ $(00 l$ and $00 \bar{l})$ to the expression in Eq. (H1).

Simulations of a tripalmitin crystallite of $100 \times 100 \times{ }_{1} N_{1,3}$ unit cells using a $180 \times 180$ grid for the powder average were performed for different thicknesses ${ }_{1} N_{1,3}$ of the crystallite. The corresponding SAXS and SANS patterns are shown 


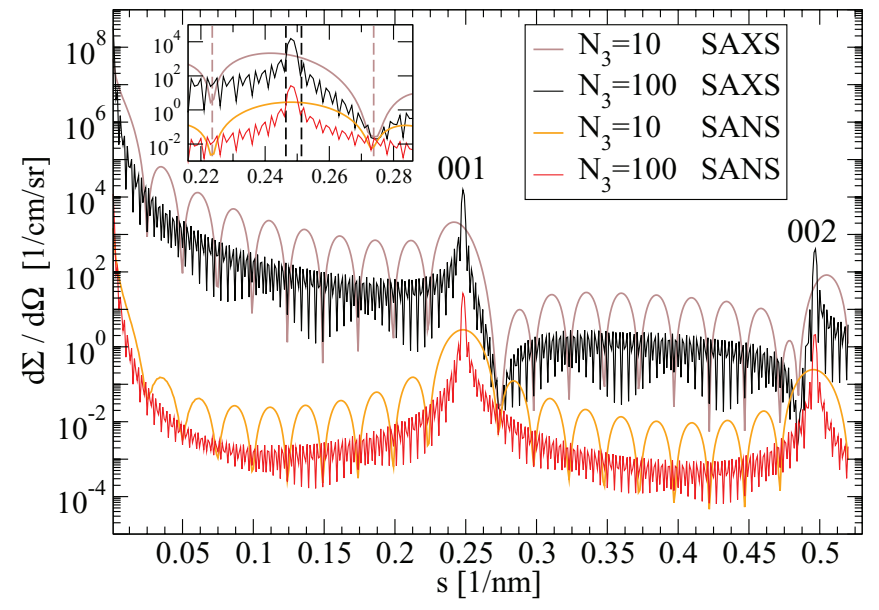

FIG. 13. (Color online) X-ray (brown and black curve) and neutron (orange and red) diffractograms for tripalmitin crystals in vacuum with different numbers of unit cells, $N_{3}$, perpendicular to the (001) planes. The integrated peak intensity for neutron scattering is nearly independent of the number of unit cells due to its slowly varying structure factor in the Bragg reflection range [cf. Fig. 14(a)] providing rather symmetric peaks. In contrast, for SAXS the integrated peak intensity converges to the theoretical value only for larger crystal sizes perpendicular to the (001) planes. The inset magnifies the $s$ range around the 001 Bragg reflection. Vertical brown and black dashed lines indicate the integration range for the 001 Bragg reflection for $N_{3}=10$ and 100, respectively, for both SAXS and SANS.

in Fig. 13 and the integrated macroscopic scattering cross sections $(d \Sigma / d \Omega)^{\text {int }}$ for the 001 Bragg reflection are tabulated in Table IV.

The agreement with the calculated values is within $10 \%$. Furthermore, the integrated peak intensity for neutron scattering is nearly independent of the number of unit cells, while for SAXS the integrated peak intensity converges to the theoretical value only for larger crystal thicknesses perpendicular to the (001) planes. This is due to the fact that the tripalmitin structure factor for neutron scattering is a slowly varying function in the Bragg reflection range [cf. Fig. 14(a)], while it has very distinct minima close to the 001 Bragg reflection position in the case of x-ray scattering.

TABLE IV. Integrated 001 Bragg reflection intensities for a tripalmitin crystallite in vacuum with different numbers of unit cells perpendicular to the (001) planes for SAXS and SANS. Good agreement is achieved between the calculated and simulated values for both, x-ray and neutron scattering in the limit of large crystal sizes.

\begin{tabular}{lccc}
\hline \hline & & \multicolumn{2}{c}{$(d \Sigma / d \Omega)^{\mathrm{int}}\left(\mathrm{cm}^{-1} \mathrm{~nm}^{-1}\right)$} \\
\cline { 3 - 4 } & $N_{3}$ & $\mathrm{X}$ rays & Neutrons \\
\hline Calculated & & 1.253 & 0.445 \\
& 2 & 11.641 & 0.422 \\
Simulated & 10 & 1.455 & 0.403 \\
& 100 & 1.198 & 0.398 \\
\hline \hline
\end{tabular}
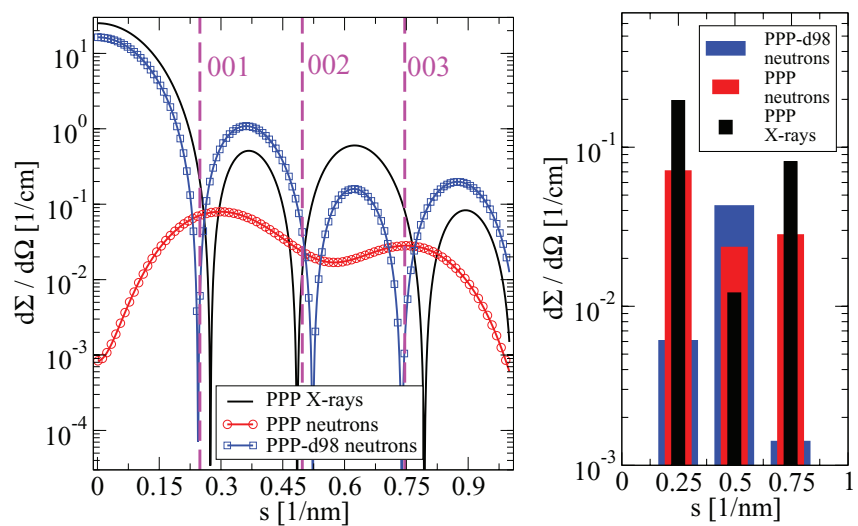

(b)

FIG. 14. (Color online) (a) X-ray and neutron structure factor $|F(s)|^{2}$ calculated in a 001 Bragg geometry for $\beta$-tripalmitin (PPP) and deuterated $\beta$-tripalmitin (PPP-d98) in vacuum in the small-angle range. The positions of the $00 l$ Bragg reflections are also marked (magenta dashed lines). While for neutron scattering the structure factor for PPP is a slowly varying function in the range of the Bragg reflections (red $\circ$ ), the sharp minima of the structure factor for X-ray scattering are close to the Bragg reflections (black line). (b) Structure factors $\left|F_{00 l}\right|^{2}=\left|F\left(s_{00 l}\right)\right|^{2}\left(s_{00 l}=l \cdot 0.2487 \mathrm{~nm}^{-1}\right)$ in the small-angle range $(l=1,2,3)$ extracted from (a). For SAXS the 001 and 003 structure factors dominate, while the 002 peak is small and often disappears in the background. For SANS the 001 and 003 peaks are considerably weakened while the 002 reflection is enhanced. The latter effect is much more pronounced for PPP-d98, where experimentally only the 002 reflection can be observed with SANS (cf. Fig. 2).

Similar results were obtained for the 002 reflection of tripalmitin or for the neutron diffraction patterns of deuterated tripalmitin.

The second test confirms again that the $\sin \vartheta$ weighting factor must be included in the powder average when using the grid algorithm in order to obtain correct Bragg reflection intensities. Without the weighting factor the simulated values were considerably larger (about 2 orders of magnitude) than the calculated ones.

\section{APPENDIX I: ESTIMATION OF THE STABILIZER THICKNESS BY VOLUMETRIC CONSIDERATIONS}

In this section, first a relationship between the distribution of the $c_{i}$, representing in the following the volume fractions of platelet ensembles with thicknesses $i$, and the corresponding number weighted distribution given by $N_{i}$ will be derived. Afterwards the total stabilizer layer thickness $d_{\mathrm{sl}}$ shall be estimated by volumetric considerations. This makes it possible to draw comparisons with the thicknesses for the stabilizer layer that were found from the fits of the SAXS and SANS data in Sec. III.

While for the simulations in Sec. II H a parallelepipedal shape of the platelets was assumed (with both lateral diameters being actually nearly the same), a disk shape is used in the following analytical calculations. Similarly, a Gaussian distribution for the platelet diameters $D$ is assumed in the simulations for the $i$ th ensemble comprising of platelets with 
$i$ layers of unit cells,

$$
\begin{gathered}
n_{i}\left(D ; \mu_{D}, \sigma_{D}\right)=\frac{N_{i}}{\sqrt{2 \pi \sigma_{D}^{2}}} \exp \left\{-\frac{\left(D-\mu_{D}\right)^{2}}{2 \sigma_{D}^{2}}\right\}, \\
N_{i}=\int_{\mathbb{R}} n_{i}\left(D ; \mu_{D}, \sigma_{D}\right) d D \approx \int_{0}^{D^{\max }} n_{i}\left(D ; \mu_{D}, \sigma_{D}\right) d D,
\end{gathered}
$$

with $N_{i}$ being the number of platelets with thickness $i$. For large enough particle diameters and not too broad distributions the integration range for the Gaussian distribution can be restricted from 0 up to a sufficiently large diameter $D^{\max }$.

Assuming disklike platelets, the volume fraction $c_{i}$ of platelets with thickness $i$ can be related to the number distribution represented by the $n_{i}$ given in Eq. (I2) by an integral over all disks with diameter $D$ and thickness $h_{i}$ (in units of $d_{001}$; cf. table in Fig. 10),

$$
h_{i}=i \cdot d_{001},
$$

weighted with $n_{i}$,

$$
c_{i}=\frac{1}{\varphi_{\text {cry }} \cdot V_{\text {tot }}} \int n_{i}\left(D ; \mu_{D}, \sigma_{D}\right)\left(\frac{\pi}{4} D^{2} h_{i}\right) d D,
$$

where $V_{\text {tot }}$ represents the volume of the dispersion and $\varphi_{\text {cry }} \cdot V_{\text {tot }}$ the total volume of the crystals in the dispersion. Equation (I4) can be rewritten with $D=\delta \cdot d_{\perp}$ as

$$
\begin{gathered}
c_{i}=\frac{N_{i} \cdot i \cdot(\pi / 4) d_{\perp}^{2} d_{001}}{\varphi_{\text {cry }} \cdot V_{\text {tot }}}\left\langle\delta_{i}^{2}\right\rangle=\frac{N_{i} \cdot i \cdot V_{u c}}{\varphi_{\text {cry }} \cdot V_{\text {tot }}}\left\langle\delta_{i}^{2}\right\rangle, \\
\left\langle\delta_{i}^{2}\right\rangle=\frac{1}{N_{i}} \int n_{i}\left(\delta ; \mu_{D} / d_{\perp}, \sigma_{D} / d_{\perp}\right) \cdot \delta^{2} d \delta,
\end{gathered}
$$

where $\quad d_{\perp}=\sqrt{4 / \pi \cdot V_{u c} / d_{001}} \quad\left(d_{\perp}=0.90 \mathrm{~nm}\right.$ for $\beta$ tripalmitin) represents a characteristic lateral length in the disk and is given as the diameter of an equivalent cylinder representing the triclinic unit cell for tripalmitin with volume $V_{u c}$ and height $d_{001}$.

For the simulations in the present study it was always assumed that the normalized diameter distribution function $n_{i}(D) / N_{i}$ is the same for all platelets independent of their thickness. This makes the $\left\langle\delta_{i}^{2}\right\rangle$ the same for all $i$. The $c_{i}$ can then be related to the $N_{i}$ [up to the constant factor in Eq. (I5)] by

$$
c_{i} \propto N_{i} \cdot i \text {. }
$$

This transformation would transform the rather pyramidal shape of the volumetric distributions represented by the $c_{i}$ as shown, e.g., in Figs. 9(a) and 9(d) to number distributions where the $N_{i}$ typically decreases with increasing platelet thickness $i$.

It should be noted that the assumption that the platelet diameters are independent of their thickness cannot be proven nor refuted since no reliable statistics of both the platelet diameters and thicknesses in such tripalmitin suspensions exist. These are difficult to obtain since in cryo-TEM the platelets preferentially orient with their large surface on the film surface [23]. Shortly after the preparation of triglyceride suspensions by high-pressure melt homogenization the nanodroplets in the hot emulsion are rather monodisperse. After recrystallization of the spherical droplets into platelets, one would therefore expect an anticorrelation of the platelet diameter with the platelet thickness so that thinner platelets possess (much) larger diameters. However, typical TEM exposures do not support this idea. Maybe those platelets that become very thin break up more easily during the recrystallization.

Using the number distribution $N_{i}$ for the platelet thicknesses under the aforementioned assumptions, a number weighted average platelet thickness (counted in numbers of unit cells) can be obtained as

$$
\langle i\rangle=\frac{\sum_{i} N_{i} \cdot i}{\sum_{i} N_{i}} \stackrel{(\mathrm{I} 7)}{=} \frac{\sum_{i} c_{i}}{\sum_{i} c_{i} / i} \stackrel{(\mathrm{D} 7)}{=}\left(\sum_{i} \frac{c_{i}}{i}\right)^{-1} .
$$

In the second part of this section, the total stabilizer layer thickness $d_{\mathrm{sl}}$ is estimated. For a suspension of monodisperse disks, $d_{\text {sl }}$ can be calculated via the relation of the volume of the stabilizer layer to the volume of the disk it covers. For a disk with diameter $D$ and height $h$ this can be written as

$$
\frac{V_{\mathrm{sl}}}{V_{\mathrm{co}}}=\frac{d_{\mathrm{sl}} S_{\mathrm{co}}}{V_{\mathrm{co}}}=\frac{d_{\mathrm{sl}}\left[2 \cdot(\pi / 4) D^{2}+\pi D h\right]}{(\pi / 4) D^{2} h},
$$

where $V_{\mathrm{sl}}$ is the volume of the stabilizer covering the disk with volume $V_{\text {co }}$ and surface $S_{\mathrm{co}} . d_{\mathrm{sl}}$ is then given as

$$
d_{\mathrm{sl}}(D, h)=\frac{V_{\mathrm{sl}}}{4 \cdot V_{\mathrm{co}}}(2 h) \frac{\frac{D}{2 h}}{1+\frac{D}{2 h}} .
$$

For a suspension of platelets with different diameters $D$ [according to the distribution function $n_{i}$ in Eq. (I1)] and thicknesses $h_{i}$ the averaged value $\left\langle d_{\mathrm{sl}}\right\rangle$ is given by

$$
\left\langle d_{\mathrm{sl}}\right\rangle=\frac{\sum_{i} \int n_{i}\left(D ; \mu_{D}, \sigma_{D}\right) \cdot d_{\mathrm{sl}}\left(D, h_{i}\right) d D}{\sum_{i} \int n_{i}\left(D ; \mu_{D}, \sigma_{D}\right) d D},
$$

which can be rewritten with Eq. (I2) and the coordinate transformation $D /\left(2 h_{i}\right)=\delta_{i}$ as

$$
\left\langle d_{\mathrm{sl}}\right\rangle=\frac{1}{\left(\sum_{i} N_{i}\right)} \frac{V_{\mathrm{sl}}}{4 \cdot V_{\mathrm{co}}} \sum_{i}\left(2 h_{i}\right) \cdot N_{i} \cdot\left\langle\frac{\delta_{i}}{\delta_{i}+1}\right\rangle .
$$

Furthermore, using Eq. (I3) and relation (I7) for replacing the $N_{i}$ with the $c_{i}$ from the fits, one finally obtains

$$
\left\langle d_{\mathrm{sl}}\right\rangle=\frac{d_{001}}{\left[\sum_{i}\left(c_{i} / i\right)\right]} \frac{V_{\mathrm{sl}}}{2 \cdot V_{\mathrm{co}}} \sum_{i} c_{i} \cdot\left\langle\frac{\delta_{i}}{\delta_{i}+1}\right\rangle .
$$

The bracketed expression involving the integral with the normalized Gaussian distribution $n_{i} / N_{i}$,

$$
\left\langle\frac{\delta_{i}}{\delta_{i}+1}\right\rangle=\frac{1}{N_{i}} \int_{0}^{\delta_{i}^{\max }} n_{i}\left(\delta ; \frac{\mu_{D}}{2 h_{i}}, \frac{\sigma_{D}}{2 h_{i}}\right) \frac{\delta_{i}}{\delta_{i}+1} d \delta_{i},
$$

can be solved numerically using sufficiently large $\delta_{i}^{\max }$.

TABLE V. Estimated stabilizer thicknesses for the polydisperse $\left(\left\langle d_{\mathrm{sl}}\right\rangle\right)$ and monodisperse model $\left(d_{\mathrm{sl}}\right)$ for the dilute tripalmitin dispersions stabilized with either DOPC or S100. For both suspensions and both models the estimated thicknesses are about 10-11 $\AA$.

\begin{tabular}{lccccc}
\hline \hline & $\begin{array}{c}\mu_{D} \\
(\mathrm{~nm})\end{array}$ & $\begin{array}{c}\sigma_{D} \\
(\mathrm{~nm})\end{array}$ & $\begin{array}{c}\left\langle d_{\mathrm{sl}}\right\rangle \\
(\AA)\end{array}$ & $\langle i\rangle$ & $\begin{array}{c}d_{\mathrm{sl}}\left(\mu_{D},\langle i\rangle \cdot d_{001}\right) \\
(\AA)\end{array}$ \\
\hline DOPC & 120 & 21.6 & 9.9 & 2.23 & 10.3 \\
S100 & 142 & 22.7 & 10.4 & 2.28 & 10.8 \\
\hline \hline
\end{tabular}


TABLE VI. Coefficients $c_{i}$ (not normalized) obtained from the simultaneous SAXS and SANS fits with model B for the suspensions with DOPC and S100 in Fig. 9.

\begin{tabular}{lccccc}
\hline \hline & $c_{1}$ & $c_{2}$ & $c_{3}$ & $c_{4}$ & $c_{5}$ \\
\hline DOPC & 0.152 & 0.267 & 0.344 & 0.214 & 0.061 \\
S100 & 0.119 & 0.320 & 0.384 & 0.174 & 0.054 \\
\hline \hline
\end{tabular}

For the dilute tripalmitin suspensions stabilized with DOPC or S100 the estimated average thicknesses $\left\langle d_{\mathrm{sl}}\right\rangle$ are tabulated in Table V. Since the dilute suspensions consist of 3\% tripalmitin and $0.79 \%$ phospholipid (cf. Sec. II B), the ratio $V_{\mathrm{sl}} / V_{\mathrm{co}}$ in Eq. (I13) can be calculated as $V_{\mathrm{sl}} / V_{\mathrm{co}}=0.79 / 3.0=0.263$ under the approximation that the weight densities of tripalmitin ( $\beta$ form at $20^{\circ} \mathrm{C}, \rho_{w}=1.037$ ) and the phospholipids [44] are close to one. Since no other experimental data were available, the $c_{i}$ in Eq. (I13) were taken from Table VI [after renormalization with Eq. (D7)]. These values were obtained from the simultaneous SAXS and SANS fits. For the normalized distribution function $n_{i} / N_{i}$ in Eq. (I14) the average diameters and polydispersities determined by the PCS measurements given in Sec. II F were used for $\mu_{D}$ and $\sigma_{D}$. The $\left\langle d_{\mathrm{sl}}\right\rangle$ are close to $11 \AA$ A for both suspensions.

Similar values for $d_{\mathrm{sl}}$ in Table V can be found with Eq. (I10) when assuming that the suspension is composed of only monodisperse disks with diameter $\mu_{D}$ and thickness $\langle i\rangle \cdot d_{001}$,

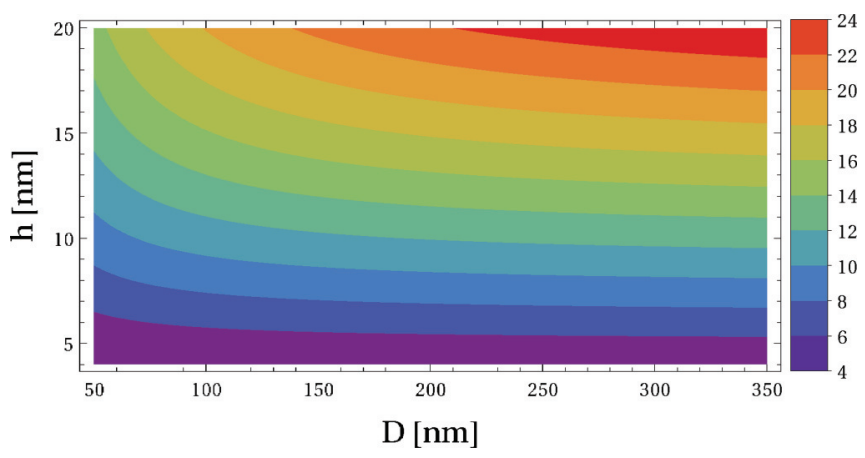

FIG. 15. (Color online) Estimated total stabilizer layer thickness (values on the color scale are given in $\AA$ ) for platelets with diameter $D$ and height $h$ according to Eq. (I10). The plot shows that for diameters in the range of 50-200 nm and thicknesses of 2-3 unit cells $(8-12 \mathrm{~nm})$ the expected total stabilizer layer thickness is typically in the range of 8-14 $\AA$.

where $\langle i\rangle$ can be obtained with Eq. (I8) using again the $c_{i}$ from the simulations.

The plot in Fig. 15 shows the range of $d_{\mathrm{sl}}$ [assuming a monodisperse system with Eq. (I10)] that can be expected for the dilute suspensions. As one can see for platelet diameters $D$ in the range of 50-200 $\mathrm{nm}$ and thicknesses $h$ in the range of $8-12 \mathrm{~nm}$ (corresponding to $2-3$ unit cells), the expected total stabilizer layer thickness is typically in the range of 8-14 $\AA$.
[1] B. Siekmann and K. Westesen, Pharm. Pharmacol. Lett. 1, 123 (1992).

[2] K. Westesen, B. Siekmann, and M. H. Koch, Int. J. Pharm. 93, 189 (1993).

[3] H. Bunjes, Curr. Opin. Colloid Interface Sci. 16, 405 (2011).

[4] K. Westesen and B. Siekmann, Int. J. Pharm. 151, 35 (1997).

[5] H. Bunjes, F. Steiniger, and W. Richter, Langmuir 23, 4005 (2007),

[6] T. Unruh, K. Westesen, P. Bösecke, P. Lindner, and M. H. J. Koch, Langmuir 18, 1796 (2002),

[7] A. Illing, T. Unruh, and M. Koch, Pharm. Res. 21, 592 (2004).

[8] J. S. Pedersen, Adv. Colloid Interface Sci. 70, 171 (1997).

[9] J. Ilavsky and P. R. Jemian, J. Appl. Crystallogr. 42, 347 (2009).

[10] http://kur.web.psi.ch/sans1/SANSSoft/sasfit.html

[11] A. Guinier, X-ray Diffraction in Crystals, Imperfect Crystals, and Amorphous Bodies (Dover, New York, 1994).

[12] T. Unruh, J. Appl. Crystallogr. 40, 1008 (2007).

[13] P. Debye, Ann. Phys. 351, 809 (1915).

[14] F. Heberle, J. Pan, R. Standaert, P. Drazba, N. Kučerka, and J. Katsaras, Eur. Biophys. J. 41, 875 (2012).

[15] H. Bunjes and T. Unruh, Adv. Drug Delivery Rev. 59, 379 (2007).

[16] T. Unruh, H. Bunjes, K. Westesen, and M. H. J. Koch, J. Phys. Chem. B 103, 10373 (1999).

[17] T. Unruh, H. Bunjes, K. Westesen, and M. H. J. Koch, Colloid Polym. Sci. 279, 398 (2001).

[18] H. Bunjes and M. H. J. Koch, J. Controlled Release 107, 229 (2005).
[19] A. J. Almeida and E. Souto, Adv. Drug Delivery Rev. 59, 478 (2007).

[20] M. Schubert and C. Müller-Goymann, Eur. J. Pharm. Biopharm. 61, 77 (2005).

[21] T. M. Göppert and R. H. Müller, Eur. J. Pharm. Biopharm. 60, 361 (2005).

[22] Z. Cui, F. Qiu, and B. R. Sloat, Int. J. Pharm. 313, 206 (2006).

[23] J. Kuntsche, J. C. Horst, and H. Bunjes, Int. J. Pharm. 417, 120 (2011).

[24] C. Loistl, Master's thesis, Fachhochschule München, 2006.

[25] T. C. Huang, H. Toraya, T. N. Blanton, and Y. Wu, J. Appl. Crystallogr. 26, 180 (1993).

[26] L. Fan, M. Degen, S. Bendle, N. Grupido, and J. Ilavsky, J. Phys.: Conf. Ser. 247, 012005 (2010).

[27] F. Zhang, J. Ilavsky, G. Long, J. Quintana, A. Allen, and P. Jemian, Metall. Mater. Trans. A 41, 1151 (2010).

[28] C. A. Dreiss, K. S. Jack, and A. P. Parker, J. Appl. Crystallogr. 39, 32 (2006).

[29] https://sourceforge.net/projects/fit2dcorr/

[30] A. P. Hammersley, S. O. Svensson, M. Hanfland, A. N. Fitch, and D. Hausermann, High Pressure Res. 14, 235 (1996).

[31] http://www.libtiff.org

[32] T. Narayanan, O. Diat, and P. B'ósecke, Nucl. Instrum. Methods Phys. Res., Sect. A 467-468, 1005 (2001).

[33] P. Boesecke, J. Appl. Crystallogr. 40, s423 (2007).

[34] A. Radulescu, V. Pipich, H. Frielinghaus, and M. S. Appavou, J. Phys.: Conf. Ser. 351, 012026 (2012).

[35] http://iffwww.iff.kfa-juelich.de/ pipich/dokuwiki/doku.php/ qtikws

[36] P. Lindner and R. Schweins, Neutron News 21, 15 (2010). 
[37] http://www.ill.eu/instruments-support/computing-for-science/ cs-software/all-software/lamp/

[38] M. Schmiele et al. (unpublished).

[39] K. Westesen and T. Wehler, J. Pharm. Sci. 81, 777 (1992).

[40] B. Siekmann and K. Westesen, in Submicron Emulsions in Drug Targeting and Delivery, edited by S. Benita (Harwood Academic, Amsterdam, 1998), pp. 205-218.

[41] M. A. Schubert, M. Harms, and C. C. Müller-Goymann, European Journal of Pharmaceutical Sciences 27, 226 (2006).

[42] H. Heiati, N. C. Phillips, and R. Tawashi, Pharm. Res. 13, 1406 (1996).

[43] https://sourceforge.net/projects/xndiff/

[44] J. F. Nagle and S. Tristram-Nagle, Biochim. Biophys. Acta, Rev. Biomembr. 1469, 159 (2000).

[45] J. Pencer, T. Mills, V. Anghel, S. Krueger, R. M. Epand, and J. Katsaras, Eur. Phys. J. E: Soft Matter Biol. Phys. 18, 447 (2005).

[46] V. F. Sears, Neutron News 3, 26 (1992).

[47] A. R. Campanelli, S. C. de Sanctis, L. Galantini, E. Giglio, and L. Scaramuzza, J. Inclusion Phenom. Mol. Recognit. Chem. 10, 367 (1991).

[48] L. Galantini, S. M. Giampaolo, L. Mannina, N. V. Pavel, and S. Viel, J. Phys. Chem. B 108, 4799 (2004).

[49] A. M. Gaspar, S. Busch, M.-S. Appavou, W. Haeussler, R. Georgii, Y. Su, and W. Doster, Biochim. Biophys. Acta, Proteins Proteomics 1804, 76 (2010).

[50] http://www.ill.eu/sites/fullprof/

[51] H. Bunjes, M. H. J. Koch, and K. Westesen, Langmuir 16, 5234 (2000).

[52] H. Bunjes, K. Westesen, and M. H. Koch, Int. J. Pharmac. 129, 159 (1996).

[53] M. Dirand, M. Bouroukba, V. Chevallier, D. Petitjean, E. Behar, and V. Ruffier-Meray, J. Chem. Eng. Data 47, 115 (2002),

[54] J. Ulmius, G. Lindblom, H. Wennerstroem, L. B. A. Johansson, K. Fontell, O. Soederman, and G. Arvidson, Biochemistry 21, 1553 (1982).

[55] S. Busch and T. Unruh, Biochim. Biophys. Acta, Biomembr. 1808, 199 (2011).

[56] F. van der Veen and F. Pfeiffer, J. Phys.: Condens. Matter 16, 5003 (2004).

[57] F. Livet, Acta Crystallogr., Sect. A: Found. Crystallogr. 63, 87 (2007).
[58] D. T. Cromer and J. T. Waber, in International Tables for X-ray Crystallography (Kynoch Press, Birmingham, 1974), Vol. 4, pp. 71-147.

[59] A. J. C. Wilson, in International Tables for X-ray Crystallography (Kluwer Academic, Dordrecht, 1995), Vol. C, pp. 500-502.

[60] http://ftp.esrf.eu/pub/scisoft/xop2.3/DabaxFiles/f0_InterTables. dat

[61] http://ftp.esrf.eu/pub/scisoft/xop2.3/DabaxFiles/DeBe_ NeutronNews.dat

[62] S. F'órster, S. Fischer, K. Zielske, C. Schellbach, M. Sztucki, P. Lindner, and J. Perlich, Adv. Colloid Interface Sci. 163, 53 (2011).

[63] M. D. Winn, C. C. Ballard, K. D. Cowtan, E. J. Dodson, P. Emsley, P. R. Evans, R. M. Keegan, E. B. Krissinel, A. G. W. Leslie, A. McCoy, S. J. McNicholas, G. N. Murshudov, N. S. Pannu, E. A. Potterton, H. R. Powell, R. J. Read, A. Vagin, and K. S. Wilson, Acta Crystallogr., Sect. D: Biol. Crystallogr. 67, 235 (2011).

[64] http://www.ysbl.york.ac.uk/ emsley/software/extras/syminfo. lib

[65] A. van Langevelde, K. van Malssen, F. Hollander, R. Peschar, and H. Schenk, Acta Crystallogr. Sect. B 55, 114 (1999).

[66] D. Chapman, Chem. Rev. 62, 433 (1962).

[67] Strictly speaking, a spherical code is a configuration (coordinates and weights) for $N$ points distributed on a unit sphere in such a way that the minimum distance between any pair of points is maximized. In the context of powder averages it is of interest to obtain configurations (crystal orientations) with a minimum number for $N$ (to reduce computation time) that still provide good simulation results.

[68] http://mathworld.wolfram.com/SpherePointPicking.html

[69] G. Marsaglia, Ann Math Stat 43, 645 (1972).

[70] http://www.bedaux.net/mtrand/

[71] M. Matsumoto and T. Nishimura, ACM Trans. Model. Comput. Simul. 8, 3 (1998).

[72] M. Bak and N. C. Nielsen, J. Magn. Reson. 125, 132 (1997).

[73] M. Mombourquette and J. Weil, J. Magn. Reson. 99, 37 (1992).

[74] A. Ponti, J. Magn. Reson. 138, 288 (1999).

[75] M. Impéror-Clerc, Interface Focus 2, 589 (2012).

[76] G. D. Wignall, D. K. Christen, and V. Ramakrishnan, J. Appl. Crystallogr. 21, 438 (1988),

[77] I. Grillo, in Soft Matter Characterization, edited by R. Borsali and R. Pecora (Springer, Netherlands, 2008), pp. 723-782. 